\title{
Senataxin controls meiotic silencing through ATR activation and chromatin remodeling
}

\author{
Abrey J Yeo ${ }^{1,2}$, Olivier J Becherel ${ }^{1,3}$, John E Luff ${ }^{1}$, Mark E Graham ${ }^{4}$, Derek Richard ${ }^{5}$, Martin F Lavin ${ }^{1,2}$ \\ ${ }^{1}$ The University of Queensland, UQ Centre for Clinical Research (UQCCR), Brisbane, QLD, Australia; ${ }^{2}$ School of Medicine, \\ The University of Queensland, Herston, Brisbane, QLD, Australia; ${ }^{3}$ School of Chemistry and Molecular Biosciences, The \\ University of Queensland, St Lucia, Brisbane, QLD, Australia; ${ }^{4}$ Children's Medical Research Institute, The University of \\ Sydney, Westmead, NSW, Australia; ${ }^{5}$ Cancer and Ageing Research Program, Faculty of Health, Queensland University of \\ Technology, Brisbane, QLD, Australia
}

Senataxin, defective in ataxia oculomotor apraxia type 2 , protects the genome by facilitating the resolution of RNA-DNA hybrids (R-loops) and other aspects of RNA processing. Disruption of this gene in mice causes failure of meiotic recombination and defective meiotic sex chromosome inactivation, leading to male infertility. Here we provide evidence that the disruption of Setx leads to reduced SUMOylation and disruption of protein localization across the XY body during meiosis. We demonstrate that senataxin and other DNA damage repair proteins, including ataxia telangiectasia and Rad3-related protein-interacting partner, are SUMOylated, and a marked downregulation of both ataxia telangiectasia and Rad3-related protein-interacting partner and TopBP1 leading to defective activation and signaling through ataxia telangiectasia and Rad3-related protein occurs in the absence of senataxin. Furthermore, chromodomain helicase DNA-binding protein 4, a component of the nucleosome remodeling and deacetylase chromatin remodeler that interacts with both ataxia telangiectasia and Rad3-related protein and senataxin was not recruited efficiently to the XY body, triggering altered histone acetylation and chromatin conformation in Setx ${ }^{-1-}$ pachytene-staged spermatocytes. These results demonstrate that senataxin has a critical role in ataxia telangiectasia and Rad3-related protein- and chromodomain helicase DNA-binding protein 4-mediated transcriptional silencing and chromatin remodeling during meiosis providing greater insight into its critical role in gene regulation to protect against neurodegeneration.

Keywords: senataxin; DNA damage repair; transcription; meiosis; chromatin remodeling

Cell Discovery (2015) 1, 15025; doi:10.1038/celldisc.2015.25; published online 29 September 2015

\section{Introduction}

Mutations in senataxin give rise to the autosomal recessive cerebellar disorder, ataxia oculomotor apraxia type 2 (AOA2) [1, 2], and also to autosomal dominant juvenile-onset, amyotrophic lateral sclerosis $4[3,4]$. It is still unclear how mutations in the Setx gene, located in close proximity, give rise to dominant and recessive disorders. However, there is evidence that posttranslational modifications of senataxin may help explain involvement in these different disease forms [5]. Senataxin is homologous to the yeast RNA-DNA

Correspondence: Martin F Lavin

Tel: +617 3346 6045; Fax: +617 3346 3973;

E-mail: m.lavin@uq.edu.au

Received 25 March 2015; accepted 17 July 2015 helicase, Sen1, a component of the Nrd1 complex, involved in RNA polymerase II transcription termination and processing of noncoding nucleolar RNAs [6-8]. Similar to that reported for other proteins mutated in cerebellar ataxias, senataxin appears to have a role in protecting the genome against DNA damage [9-11]. Greater insight into its role in the response to DNA damage has been provided in recent studies. This protein was reported to interact with proteins involved in transcription and was shown to have a role in transcription termination, mRNA splicing efficiency and splice site selection [12]. Insight into its role in transcriptional termination was provided by the observation that senataxin resolves RNA-DNA hybrids (R-loops) and as a consequence allows Xrn2 $\left(5^{\prime}>3^{\prime}\right.$ exonuclease) access at the $3^{\prime}$ cleavage poly (A) site, nascent transcript degradation and consequently 
polymerase II release and termination [13]. In yeast, Sen1 has also been shown to protect the genome from R-loop-mediated DNA damage [14]. The continuing presence of R-loops can have a negative impact on transcription elongation, leading to collisions with DNA replication forks and/or compromising genomic integrity by being a source of hypermutation or causing hyper-recombination [15]. More recently, senataxin has been localized to sites of collision between components of the replisome and the transcriptional apparatus [16]. By recognizing and resolving R-loops, it has an important role at the interface of transcription and the DNA damage response [16]. Evidence for this also exists in yeast where Sen1 has a key role in coordinating replication and transcription [17]. Senataxin has also been shown to suppress the anti-viral transcriptional response to control viral biogenesis [18]. It appears to do so by negatively regulating IRF3-dependent expression and promoting early termination of RNA polymerase II. At present, it remains unclear as to whether R-loops are responsible for the neurodegenerative changes that characterize AOA2. We previously reported the accumulation of R-loops in proliferating cells of Setx mutant mice treated with the topoisomerase inhibitor, topotecan hydrochloride, but failed to detect any R-loops in the brains of mice treated with the same agent [19].

Disruption of the Setx gene in mice failed to reveal any significant neurological abnormalities or any evidence of neurodegeneration [20]. However, male mutant mice were infertile and there was evidence of reduced fertility in females. Male germ cells proceeded normally from spermatogonia up to the pachytene stage of meiosis in Setx $x^{--}$, but failed to form mature spermatids [20]. Successful generation of programmed DNA double-strand breaks (DSB) and the initiation of DNA repair was observed in Setx ${ }^{-/}$spermatocytes, but RAD51 foci were abnormally retained in the pachytene stage and DNA DSBs persisted, revealing a defect in homologous recombination. In addition, absence of Mlh1 foci in Setx $^{-/-}$spermatocytes provided further evidence of failure to complete recombination. Set ${ }^{-/-}$spermatocytes also showed an accumulation of R-loops and underwent apoptosis. Furthermore, senataxin localized to the $\mathrm{XY}$ chromosomes at the pachytene stage and abnormal diffusion of DNA damage rep (DDR) proteins into the $\mathrm{XY}$ chromatin domain was observed, predicting that the XY chromosomes would fail to be transcriptionally silenced in Set $x^{-/-}$pachytene cells, which turned out to be the case [20]. These results pointed to a key role for senataxin in both meiotic homologous recombination and in meiotic sex chromosome inactivation (MSCI).

In this study, we used the Setx mutant mouse model to investigate the role of senataxin in meiotic transcriptional silencing. Loss of senataxin caused a marked decrease in protein SUMOylation across the $\mathrm{XY}$ body during the pachytene stage of meiosis. Senataxin itself was SUMOylated as well as several other proteins that include the ataxia telangiectasia and Rad3-related protein (ATR)-interacting partner, ATRIP, and in its absence, there was a marked downregulation and mislocalization of both ATRIP and TopBP1. Downregulation of ATRIP resulted from the failure of NIMA (never in mitosis gene a)-related kinase 1 (NEK1) to localize to the XY body and this led to defective ATR signaling. Chromodomain helicase DNA-binding protein 4 (CHD4), a key component of the nucleosome remodeling and deacetylase (NuRD) histone deacetylase chromatin remodeling complex, which interacts with both ATR and senataxin, was not efficiently recruited to the XY body, leading to persistent histone acetylation of the XY chromosomes and altered chromatin structure in Set $x^{-/}$spermatocytes. These data provide further evidence of a critical role for senataxin in transcriptional silencing.

\section{Results}

\section{Senataxin is SUMOylated on the XY body}

The various stages of meiosis can be differentiated by immunostaining for synaptonemal complex protein 3 (SCP3), a proteinaceous component of the axial element of the synaptonemal complex required for chromosomal synapsis [21]. We have previously shown that senataxin localized as a diffuse 'cloud' to the XY chromosomes in spermatocytes at the pachytene stage of male meiosis where it has an essential but undefined role in MSCI [20] (Figure 1a). Small ubiquitin-like modifier (SUMO) proteins have also been localized to the XY chromosomes and they have been implicated in the initiation of MSCI [20]. A yeast two-hydrid screen to detect SUMO-1- and SUMO-2/3-interacting motifs previously identified proteins involved in DNA repair and transcriptional repression [22]. and growing evidence implicated SUMO as an important posttranslational modification to modulate protein assemblies, activity and function [23]. Therefore, we investigated whether senataxin might be SUMOylated as part of its role in MSCI. Immunostaining for SUMO-1 of spermatocyte spreads revealed an intense, diffuse pattern of staining across the $\mathrm{XY}$ body in 
$\operatorname{Set}^{+/+}$spermatocytes (Figure 1b). On the other hand, when senataxin was disrupted, staining for SUMO-1 was significantly reduced and was confined to the unsynapsed region of the axial element of the XY chromosomes. A similar pattern of staining was observed with SUMO-2/3 (Supplementary Figure S1A).

a
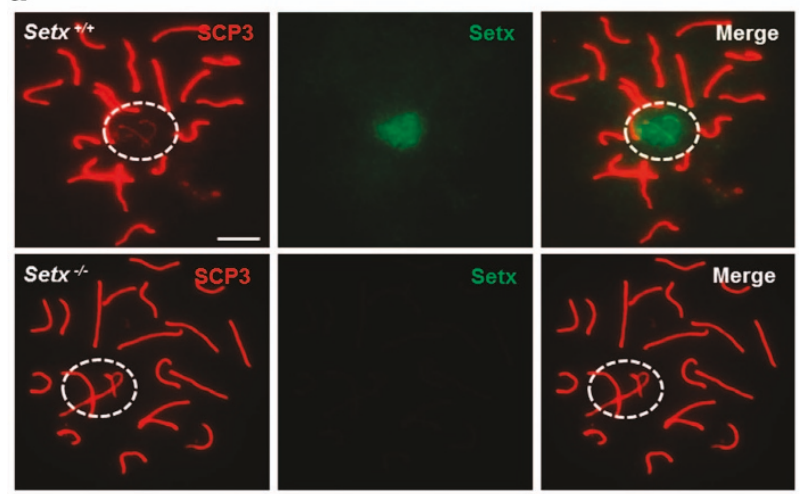

b
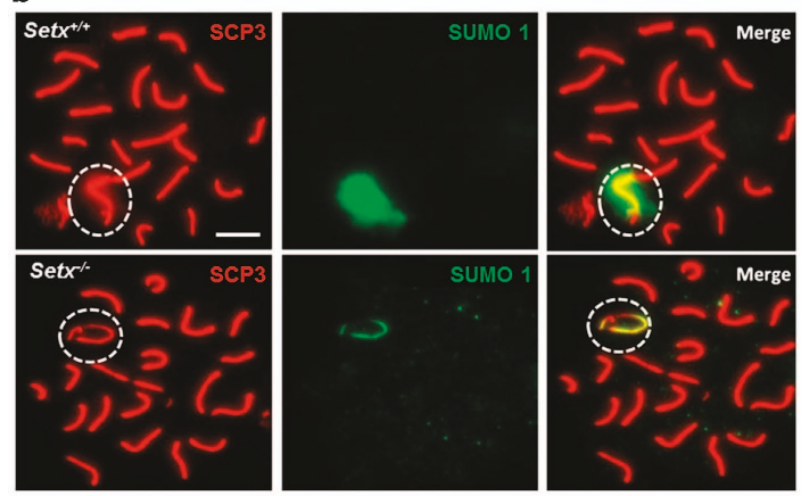

c

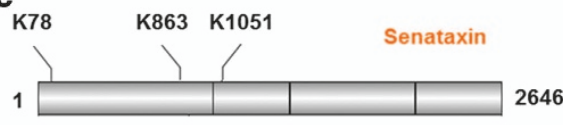

d
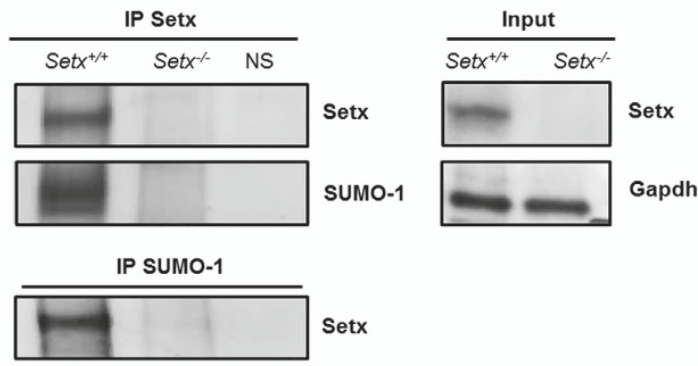

e
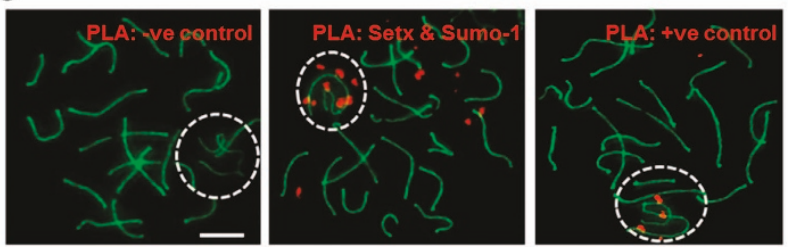

The early appearance of SUMO-1, which precedes the accumulation of $\gamma \mathrm{H} 2 \mathrm{AX}$ on the $\mathrm{XY}$ chromosomes, and the lack of SUMO-2/3 detection in zygotene spermatocytes, suggested a more specific role for SUMO-1 in the initiation of XY inactivation [24]. Therefore, SUMO-1 was chosen for further investigation. A bioinformatics tool, GPS-SUMO (http: //sumosp.biocuckoo.org), which predicts sites of SUMOylation and SUMO-interacting motifs based on consensus sequence predicted three sites of SUMOylation (Figure 1c) and three SUMO-interacting motifs in senataxin (Supplementary Figure S1B). All three potential SUMOylation sites are located in the $\mathrm{N}$ terminus of senataxin. Consistent with this prediction, Hecker et al. [22] previously identified senataxin as a SUMO-1/2-interacting protein using a yeast twohybrid approach, and more recently an interaction between senataxin and Rrp45, a subunit of the exosome, was found to be dependent on the SUMOylation of senataxin [5]. Co-immunoprecipitation of senataxin and SUMO-1 was detected in cell extracts made from Set $x^{+/+}$testes, and as expected, no signal was detected in Set $^{-/-}$extracts (Figure 1d). This was confirmed

Figure 1 Senataxin is SUMOylated at the XY chromosomes in spermatocytes. (a) Immunostaining for senataxin revealed its localization to the $X Y$ chromosomes as a diffuse cloud during the pachytene stage in spermatocytes of Set $x^{+/+}$mice. Some background staining was also observed on the autosomes. As expected, no senataxin was detected in Setx ${ }^{-/}$spermatocytes. (b) Immunostaining for SUMO-1 in Setx ${ }^{+/+}$and Setx ${ }^{-/}$spermatocytes revealed a diffuse staining over the $X Y$ chromosomes in Set $x^{+/+}$spermatocytes. In contrast, staining was largely restricted to the axes of the XY chromosomes in Setx ${ }^{-/-}$spermatocytes. (c) GPS-SUMO, a bioinformatics tool, predicted three potential SUMOylation sites for senataxin. (d) Endogenous senataxin and SUMO-1 were co-immunoprecipitated and immunoblotted separately, showing SUMOylation of senataxin in Setx ${ }^{+/+}$ spermatocytes. A negative control for the immunoprecipitation (IP) using nonspecific (NS) immunoglobulin G (IgG) was also performed. As expected, input lanes show the presence of senataxin in Setx ${ }^{+/+}$but not in Setx ${ }^{-/-}$total cell extracts. Glyceraldehyde 3-phosphate dehydrogenase (GAPDH) was used as a loading control. (e) Through a proximity ligation assay (PLA), an interaction between senataxin and SUMO-1 was observed over the $\mathrm{XY}$ chromosomes as a concentration of red foci (middle panel). Some more scattered red foci were also observed over the autosomes. A negative control without a primary antibody (left panel) as well as a positive control (right panel) using antibodies against breast cancer type 1 susceptibility protein (BRCA1) and ataxia telangiectasia and Rad3-related protein (ATR), two proteins known to interact directly, was performed alongside. Scale bar, $20 \mu \mathrm{m}$. Synaptonemal complex protein 3 (SCP3), which indicates the axial elements of the synaptonemal complex. Dotted circle, XY chromosomes. 
a

\begin{tabular}{cccccccc} 
Peptide sequence & $\begin{array}{c}\text { Mascotlon } \\
\text { score }\end{array}$ & & $\begin{array}{c}\text { p value } \\
\text { mass }(\mathbf{m} / \mathbf{z})\end{array}$ & Charge & $\begin{array}{c}\text { Peptide } \\
\text { mass (Da) }\end{array}$ & $\begin{array}{c}\text { Mass error } \\
\text { (ppm) }\end{array}$ & $\begin{array}{c}\text { Sequence } \\
\text { Numbers }\end{array}$ \\
\hline R/EAQNLAFTGLNL & 76.8 & 0.003 & 808.94 & 2 & $1,615.87$ & 2.1 & $378-392$ \\
$\quad$ VAR/T & & & & & & & \\
R/KGSSVVLK/S & 32.8 & 0.021 & 409.26 & 2 & 816.51 & -1.5 & $227-234$ \\
R/NLSSAHK/V & 36.5 & 0.005 & 378.70 & 2 & 755.39 & 2.2 & $81-87$
\end{tabular}

ATR-interacting protein, 85,548.0 Da, ATRIP_MOUSE, Uniprot entry: Q8BMG1 3 exclusive unique peptides, $30 / 785$ amino acids ( $4 \%$ coverage)

\begin{tabular}{|c|c|c|c|}
\hline$M A G T P A P N S H$ & $R K Q S G G L E P F$ & $P G L S R S I E N F$ & $P S K R A R$ \\
\hline$T T \vee P D P E D P F$ & $G E H A E F T A D D$ & $L E E L D \mid L A S Q$ & $A L S Q C P \vee A P$ \\
\hline$N L S S A H K \vee R R$ & $L D G L P N S P \mid R$ & KSREDIPVKD & $N F E L E V L Q \mid Q$ \\
\hline$E L K E K L K A$ & $M E E E I L I K N G$ & E I K I LRDSLR & $Q T E S V L E E Q$ \\
\hline$H F L L E Q E K$ & $T Q A L S E K E K E$ & $F S R K L Q S L Q S$ & $E L Q F K D A E M$ \\
\hline$S Q S N G$ & $R T N K P A A P S V$ & $S H V S P R K G S S$ & V V L K SE \\
\hline $\mathrm{T} \mathrm{K}$ & ESF $S A N T P L F$ & $H P C Q T E A G H R$ & $F L \vee G Q E \vee S D N$ \\
\hline HS L G G & $K Q D \vee Q Q R । L A$ & $D G W M Q R K D A Q$ & GS I L INLL L K \\
\hline$Q P L \vee P G S S L G$ & LCHLLSSCPE & $\vee P T G T L L Q P P$ & $G L S T L P G T S$ \\
\hline$L R T$ I S S S D GP & $F S P S A L R E A Q$ & $N L A F T G L N L V$ & A R T E S S H \\
\hline$M A G R R \vee F P L H$ & $Q L P G A V H L L P$ & $L \vee Q F F \vee G L H C$ & $Q A L Q D L A P A K$ \\
\hline$K S G \vee P G D S A T$ & $H T S C M S S G \vee E$ & A S P EDS I HGL & $E S F S \vee A S L S V$ \\
\hline $\mathrm{L} Q \mathrm{QNL} \vee \mathrm{CH}$ & $\vee \vee C L L L S G M G$ & $T E A A A R E G N L$ & $V Q T C A D$ \\
\hline$S R E D A$ & $H P L L K M L L Q L$ & $S S A A$ & $F Q A S V L G L C$ \\
\hline L V K & $S S D L L P R F S C$ & $\vee F P \vee L P Q C L G$ & $S A L P L P C \vee L L$ \\
\hline$A \vee E L L S \vee L L D$ & $H D S L A W Q L C S$ & HP E G C L L L R L & $Y M Y \perp T S R P D R$ \\
\hline$S E T Q W L Q L$ & $E Q E V \vee W L L A K$ & $L S \vee Q S P A P A G$ & I GSDCHCNVE \\
\hline$A \vee R A L T \vee M L H$ & $R Q W L T \vee R R A G$ & $G P R T H Q Q K Q T$ & I RCLRDTVLL \\
\hline L H S L S Q K D & F T VHCVEVLH & $Q Y D Q \vee M P G \vee S$ & $M L I$ \\
\hline & & & \\
\hline
\end{tabular}

C A A E T DLEDS

EM D C N

b IP sumo-1

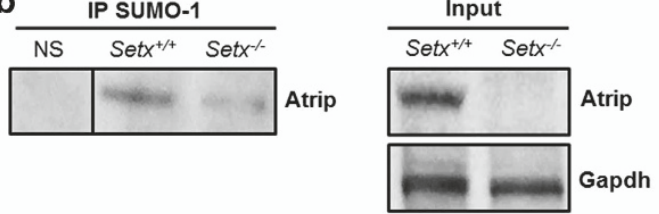

C

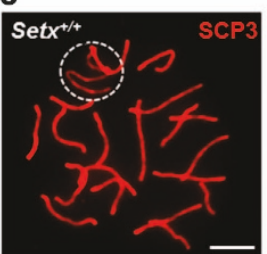

Setx

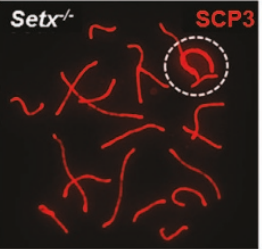

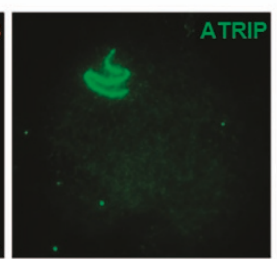

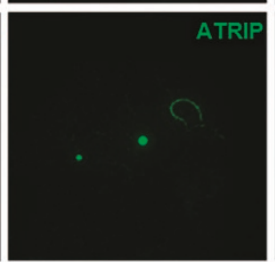

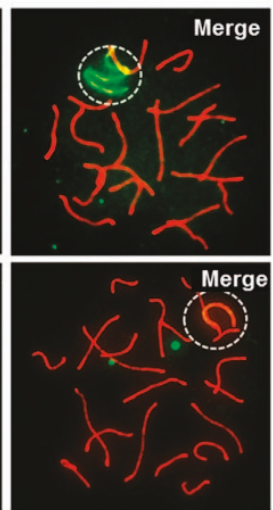

d

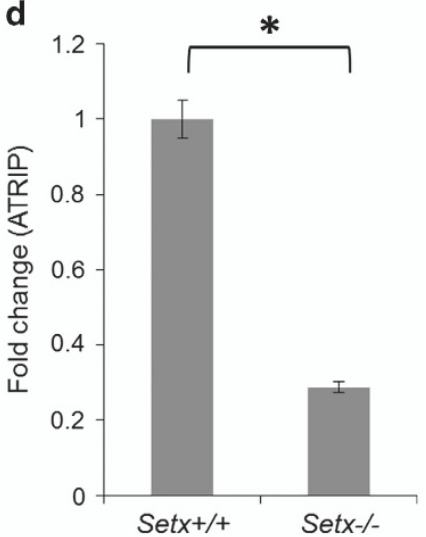

Figure 2 Ataxia telangiectasia and Rad3-related protein-interacting partner (ATRIP) protein levels are reduced in the absence of senataxin. (a) Liquid chromatography mass spectrometry (LC-MS/MS) on immunoprecipitation (IP) from Setx ${ }^{+/+}$and Setx ${ }^{-/-}$

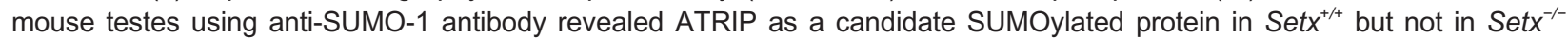
spermatocytes. Protein identification was visualized on the Scaffold Q+S application (Proteome Software, Portland, OR, USA). (b) IP from Setx ${ }^{+/+}$and Setx ${ }^{-/-}$testes extracts for SUMO-1 and immunoblotting for ATRIP confirmed the SUMOylation of ATRIP. A negative control for the IP using nonspecific (NS) immunoglobulin G (IgG) was also performed. Input lanes show the presence of ATRIP in Setx ${ }^{+/+}$but not in Setx ${ }^{-/-}$total cell extracts. Glyceraldehyde 3-phosphate dehydrogenase (GAPDH) was used as a loading control. (c) Immunostaining for ATRIP revealed a strong signal on the axial element of the XY chromosomes in Setx ${ }^{+/+}$ spermatocytes. On the other hand, decreased staining intensity for ATRIP was observed in Setx ${ }^{-/}$spermatocytes. Scale bar, $20 \mu \mathrm{m}$. SCP3, Synaptonemal complex protein 3. Dotted circle, XY chromosomes. (d) Quantitation of ATRIP fluorescence intensity staining (shown as fold change) in Setx ${ }^{+/+}$and Setx ${ }^{-/-}$. Data are plotted as the mean \pm S.d. Statistical analysis was performed using the Student's $t$-test, ${ }^{*} P<0.05$. Number of animals, $n=3$. 
by the immunoprecipitation with an anti-SUMO-1 antibody (Figure 1d). Although immunoprecipitation confirmed the SUMOylation of senataxin, a proximity ligation assay (PLA) for both senataxin and SUMO-1 on $\operatorname{Setx}^{+/+}$spermatocyte spreads was performed to determine the localization of the senataxin/SUMO-1 interaction at the pachytene stage. PLA allows the visualization of in situ endogenous protein-protein interactions by the appearance of fluorescent spots because of amplification and subsequent incorporation of fluorescent nucleotides on complementary strands that are conjugated to target antibodies [21]. The results provided further evidence that senataxin and SUMO-1 interacted with one another primarily on the $\mathrm{XY}$ chromosomes, as evidenced by the majority of the positive signals (red dots) focused over the XY body (Figure 1e). These results demonstrate that senataxin is SUMOylated and that it localizes primarily to the XY chromosomes.

Defective SUMOylation of XY chromosome proteins in Set $x^{-l-}$ pachytene cells

Given that the SUMOylation signal intensity was markedly decreased over the XY body and was mislocalized in the absence of senataxin, we predicted that other proteins localized to the XY body would depend on senataxin for their SUMOylation. To identify these SUMOylated proteins, we used immunoprecipitation with an anti-SUMO-1 antibody with extracts from $\operatorname{Setx}^{+/+}$and $\operatorname{Setx}^{-/-}$testes followed by sodium dodecyl sulfate-polyacrylamide gel electrophoresis (SDS-PAGE), and identification of Coomassie-stained bands by liquid chromatography mass spectrometry (LC-MS/MS). A stringent threshold for protein identification was set at $P<0.01$, the false discovery rate was set at $0.01 \%$ and a minimum of two unique peptides was used as an arbitrary threshold for confident protein identification. Several proteins were identified (Supplementary Table S1), which included several candidates related or potentially related to defective SUMOylation.

Among the SUMOylated proteins or those bound to SUMOylated proteins were those involved in chromatin remodeling (ATRX and RUVB2), in transcription regulation (DHX15 and YBOX3) and the regulatory partner of ATR, ATRIP, consistent with a role for SUMOylation in the DNA damage response and transcription. Data for ATRIP identification are presented in Figure 2a. We confirmed the SUMOylation of ATRIP in spermatocytes by carrying out immunoprecipitation with anti-SUMO-1 antibody and immunoblotting with anti-ATRIP antibody
(Figure 2b). A reduction in ATRIP SUMOylation was observed in Setx ${ }^{-/-}$spermatocytes as compared with Set $x^{+/+}$, suggesting a lower level of ATRIP in Setx ${ }^{-/-}$. Indeed, this is in agreement with the difficulty of detecting endogenous levels of ATRIP in total cell extracts from Setx ${ }^{-/-}$(Figure 2b). Furthermore, the decrease in ATRIP protein levels observed in Setx $x^{-1-}$ testes was not a defect in the expression of ATRIP (Supplementary Figure S3B), suggesting that senataxin does not directly affect the transcriptional regulation of ATRIP.

The reduction of ATRIP, and consequently SUMOylated ATRIP, in Setx $x^{-/}$spermatocytes and the importance of ATRIP in maintaining the stability and activity of ATR prompted us to investigate the localization of ATRIP in spermatocytes from these mice. A strong signal for ATRIP that was restricted largely to the axial element of the XY chromosomes was observed in $\operatorname{Setx}^{+/+}$pachytene spermatocytes (Figure 2c). Additionally, some background staining for ATRIP was observed on the autosomes. Although the localization of ATRIP in Setx ${ }^{-/-}$spermatocytes was similar to that observed in Setx ${ }^{+/+}$spermatocytes, the signal was markedly reduced. Thus, a reduction in ATRIP protein levels and/or defective recruitment to the XY chromosomes appears to occur in Setx ${ }^{-/}$ pachytene-staged spermatocytes. Quantitation of ATRIP fluorescence intensity staining on the XY chromosomes revealed an $\sim 3.3$-fold reduction in $\operatorname{Setx}^{-/-}$compared with $\operatorname{Setx}^{+/+}$(Figure 2d).

\section{Activation of ATR and downstream signaling is defective in Setx ${ }^{-1-}$ spermatocytes}

ATR has also been shown to have a key role in MSCI where it is required for the phosphorylation of $\mathrm{H} 2 \mathrm{AX}$ [25]. Briefly, ATR is a kinase that is activated upon recognition of DNA damage, which includes single-stranded DNA breaks [26]. Single-stranded DNA is first detected and coated by replication protein A (RPA), and subsequently the ATR-ATRIP complex is recruited to sites of DNA damage and activated by TopBP1, leading to the activation/ phosphorylation of its downstream substrates, which include RPA and checkpoint kinase 1 (CHK1) (Figure 3a) [27, 28]. The initiation of MSCI begins with the recruitment of breast cancer type 1 susceptibility protein (BRCA1) to the axes of the XY chromosomes, which then promotes ATR recruitment [25]. As shown in Supplementary Figure S2, normal recruitment of BRCA1 was observed in both $\operatorname{Set}^{+/+}$and Setx ${ }^{-/-}$mice, indicating that the initiation of MSCI occurs typically in these mice. This then suggested that the defect in 


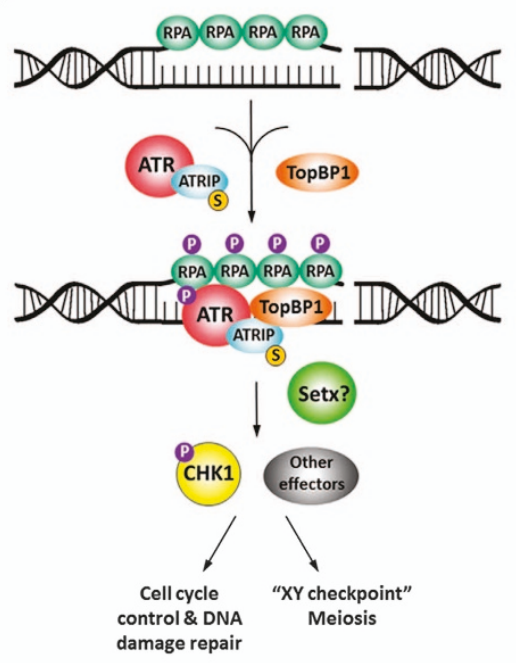

b
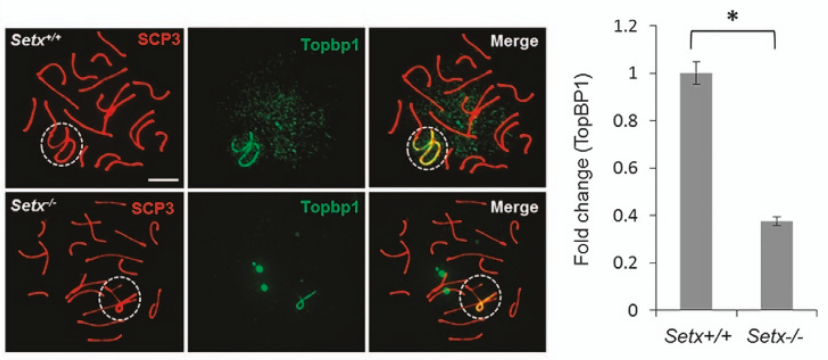

C
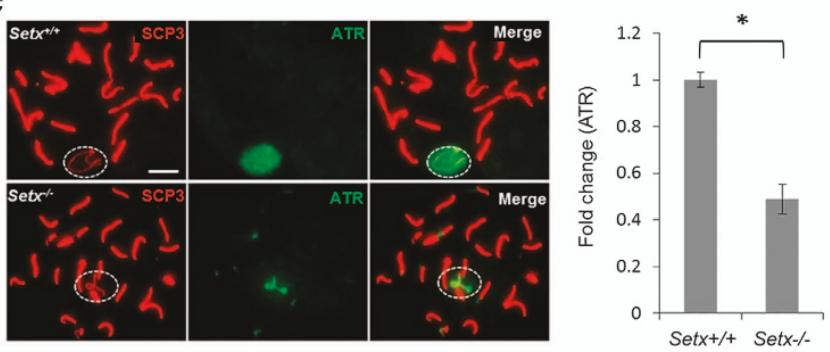

d

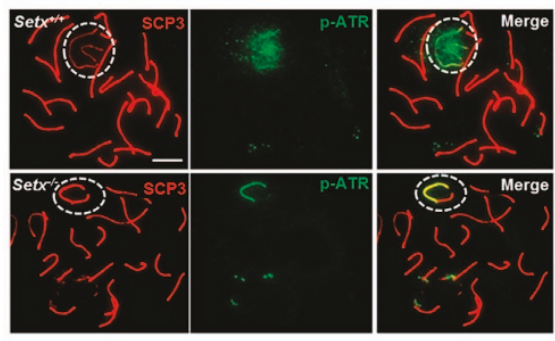

f

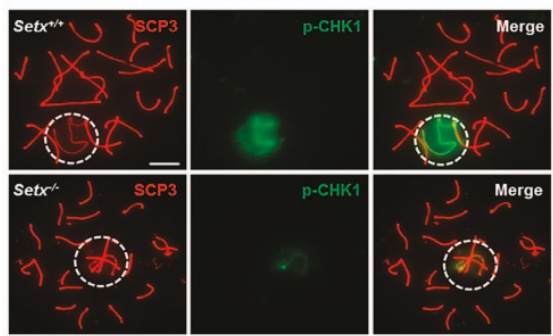

e
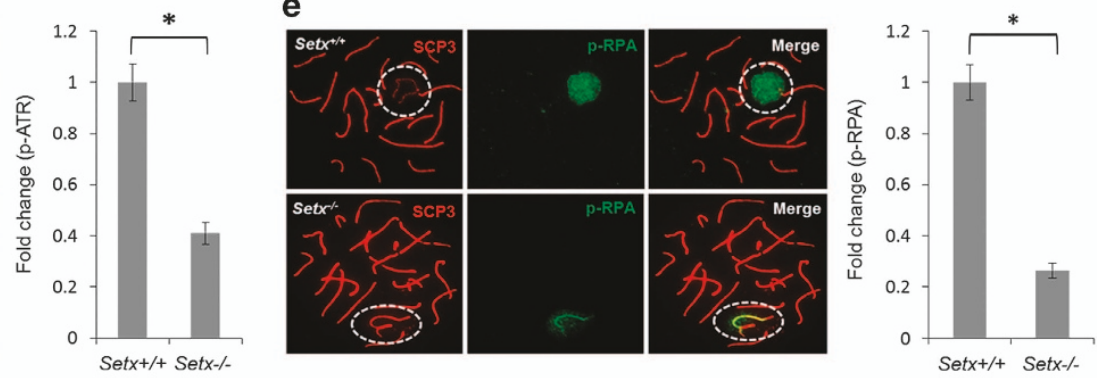

Figure 3 Ataxia telangiectasia and Rad3-related protein (ATR) activity and signaling is defective in the absence of senataxin. (a) A schematic illustration of the ATR signaling pathway and the proteins investigated. (b) TopBP1 was observed to localize predominantly to the axial element of the $\mathrm{XY}$ chromosomes in Setx ${ }^{+/+}$spermatocytes. In contrast, decreased staining intensity for TopBP1 was observed in Setx ${ }^{-/-}$spermatocytes. Quantitation of TopBP1 fluorescence intensity staining (shown as fold change) in Setx ${ }^{+/+}$and Setx ${ }^{-/-}$. (c) ATR localized as a cloud in Setx ${ }^{+/+}$spermatocytes, whereas in Setx ${ }^{-/-}$, ATR was confined to the axial element of the $X Y$ chromosomes. Quantitation of ATR fluorescence intensity staining (shown as fold change) in Setx ${ }^{+/+}$and Setx $^{-/}$. (d) Immunostaining for phospho-S428 ATR (p-ATR(S428) showed a cloud over the XY chromosomes in Setx ${ }^{+/+}$ spermatocytes as expected, whereas phospho-S428 ATR (p-ATR) was retained on the axial element of the XY chromosomes in Setx ${ }^{-/-}$spermatocytes. Quantitation of p-ATR fluorescence intensity staining (shown as fold change) in Setx ${ }^{+/+}$and Setx ${ }^{-/}$. (e) Immunostaining for p-RPA(S33) showed a diffuse staining pattern over the XY body in Setx ${ }^{+/+}$spermatocytes as expected, whereas it was largely retained on the axial element of the XY chromosomes in Setx ${ }^{-/-}$spermatocytes. Quantitation of p-RPA fluorescence intensity staining (shown as fold change) in Setx ${ }^{+/+}$and Setx ${ }^{-/}$. (f) Localization of phospho-S317 CHK1(S317) (p-CHK1(S317)) to XY chromatin in Setx ${ }^{+/+}$. Reduced levels of p-CHK1(S317) localized mainly to the axial element of the XY chromosomes in Setx ${ }^{-/}$spermatocytes. Quantitation of p-CHK1 fluorescence intensity staining (shown as fold change) in Setx ${ }^{+/+}$ and Setx ${ }^{-/}$. Scale bar, $20 \mu \mathrm{m}$. Synaptonemal complex protein 3 (SCP3). Dotted circle, XY chromosomes. All data were plotted as the mean \pm s.d. Statistical analysis was performed using the Student's $t$-test, ${ }^{*} P<0.05$. Number of animals, $n=3$. 
MSCI in Setx $^{-/-}$spermatocytes lies downstream of this initial step. As TopBP1 acts as a general activator of ATR [22] and this activation is mediated through ATRIP, we predicted that there might also be a defect in ATR activation and downstream signaling in Setx $x^{-/-}$ spermatocytes. Immunostaining on spermatocytes of Setx mice showed that TopBP1 localized predominantly on the axial elements of the XY chromosomes in $\mathrm{Setx}^{+/+}$spermatocytes as expected, whereas a much fainter signal was observed in Setx $x^{-/-}$spermatocytes, despite a similar localization to that observed in Set $x^{+/+}$(Figure 3b). Quantitation of fluorescence intensity of TopBP1 also showed an $\sim 2$.6-fold reduction in $\operatorname{Set}^{-/-}$as compared with $\operatorname{Set}^{+/+}$(Figure 3b). These results suggested a potential defect at the level of ATR function and downstream signaling in the absence of senataxin. In addition to coordinating DDR, ATR is required for the phosphorylation of histone $\mathrm{H} 2 \mathrm{AX}$ on the XY chromosomes during MSCI [25]. Although the exact purpose of this phosphorylation remains unclear, it has been hypothesized that it is necessary for initiating chromatin condensation and repression of transcription [29]. We observed that ATR formed a diffuse cloud over the XY body in Set $x^{+/+}$ spermatocytes as reported previously [30], whereas it was localized largely on the axial element of the XY chromosomes in $\operatorname{Setx}^{-/-}$spermatocytes, failing to diffuse out into XY chromatin (Figure 3c). Quantitation of fluorescence intensity of ATR also showed an $\sim 2$-fold reduction in $\operatorname{Set}^{-/-}$as compared with $\operatorname{Set}^{+/+}$ (Figure 3c). To determine whether the altered localization of ATR might reflect a difference in its activity, immunostaining against an active form of ATR, phospho-S428 ATR (p-ATR) was performed. Results showed a similar staining pattern to that observed for ATR, with staining being confined to the unsynapsed region of the axial elements of the XY chromosomes (Figure 3d). Quantitation of fluorescence intensity of p-ATR also showed an $\sim 2$.4-fold reduction in Setx ${ }^{-/-}$ as compared with $\operatorname{Set}^{+/+}$(Figure 3d). These data point to a defect in the diffusion of p-ATR, which could lead to alterations in the downstream DDR signaling that occurs during MSCI. To examine this possibility, we next investigated ATR signaling in the absence of senataxin by immunostaining for two ATR substrates, RPA and CHK1 [31, 32]. Phospho-S33 RPA (p-RPA) was observed to form an intense diffuse cloud over the XY chromosomes in Set $x^{+/+}$spermatocytes, whereas p-RPA was significantly reduced in intensity and localized mainly on the axial element of the XY chromosomes in $\operatorname{Set}^{-/-}$spermatocytes (Figure 3e). Quantitation of fluorescence intensity of p-RPA also showed an $\sim 2.2$-fold reduction in $\operatorname{Set}^{-/-}$as compared with $\operatorname{Set}^{+/+}$(Figure 3e). CHK1, a kinase required for cell cycle checkpoint activation and a well-documented downstream target of ATR for phosphorylation $[33,34]$, was next assessed. The ATR-phosphorylated form of CHK1 phospho-S317 CHK1 (p-CHK1) formed a diffuse cloud over the XY chromosomes in Set ${ }^{+/+}$spermatocytes, whereas a reduced signal that was largely retained on the axial element was observed in Setx $^{-/-}$spermatocytes (Figure 3f). Quantitation of fluorescence intensity of p-CHK1 also showed an $\sim 3.8$-fold reduction in $\operatorname{Set}^{-/-}$as compared with Set $^{+/+}$ (Figure 3f). Both p-RPA and p-CHK1 exhibited similar staining patterns as those of ATR and p-ATR in Setx ${ }^{+/+}$and Setx $x^{-/}$spermatocytes. Immunoblotting was carried to determine the levels of the proteins involved in ATR signaling. Both ATRIP and TopBP1 protein levels were markedly reduced in $\operatorname{Set}^{-/-}$, in agreement with the immunofluorescence data (Supplementary Figure S3A). However, similar levels of TopBP1 and ATRIP mRNAs were observed between $\mathrm{Set}^{+/+}$and $\mathrm{Set}^{-/-}$, suggesting that senataxin does not directly regulate the expression of these genes at the transcriptional level (Supplementary Figure S3B). Although ATR and CHK1 were present in normal levels, there was evidence that ATR activation was lower in $\mathrm{Setx}^{-l-}$ as observed with decreased p-ATR protein levels (Supplementary Figure S3A). Taken together, these data support a defect in ATR activity and downstream signaling in $\operatorname{Setx}^{-/-}$as this activity is confined to the axial elements of the XY chromosomes, which could account for the failure of MSCI in these mice.

A recent report showed that the kinase activity of NEK1 is critical for initiating the ATR response following DNA damage [35]. Cells lacking NEK1 failed to support ATR autophosphorylation, did not efficiently phosphorylate ATR downstream substrates and exhibited significantly reduced levels of ATRIP [35]. Thus, it is possible that in $\operatorname{Setx}^{-/-}$cells, a defect in NEK1 may be present, which could in turn account for the defect in ATR activity observed here, and consequently a failure in MSCI. Immunoblotting for NEK1 protein from total cell extracts from testes of Set $^{+/+}$and Setx ${ }^{-/-}$mice revealed an $\sim 1.7$ fold decrease in NEK1 in $\operatorname{Set}^{-/-}$(Figure 4a), despite similar gene expression levels (Supplementary Figure S3B). Immunostaining showed that NEK1 localized to the axial element of the autosomes, with a stronger signal on the XY chromosomes in $\operatorname{Set}^{+/+}$spermatocytes (Figure 4b). In contrast, NEK1 localized neither to the autosomes nor the XY chromosomes in Setx ${ }^{-1-}$ 
a
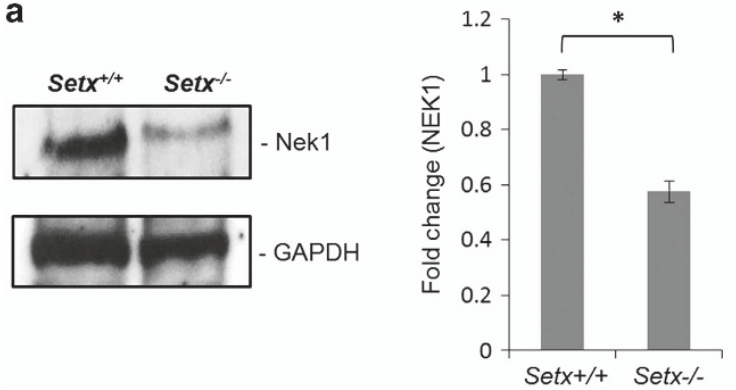

b
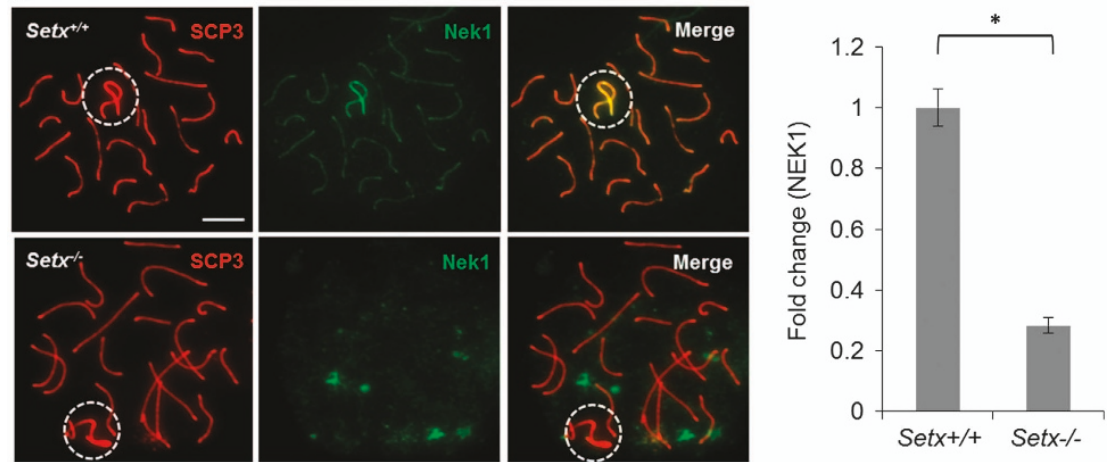

Figure 4 NNIMA (never in mitosis gene a)-related kinase 1 (NEK1) localization and protein levels are disrupted in the absence of senataxin. (a) Immunoblotting from testes extracts revealed a decrease in NEK1 protein levels in Setx ${ }^{-/-}$as compared with Setx ${ }^{+/+}$ spermatocytes. $\alpha$-Tubulin was used as a loading control. Quantitation of NEK1 band intensity staining (shown as fold change) in Setx $^{+/+}$and Setx ${ }^{-/-}$. (b) NEK1 was observed to localize specifically to the axial element of the autosomes with a stronger intensity on the XY chromosomes in Setx ${ }^{+/+}$spermatocytes, whereas NEK1 in Setx ${ }^{-/}$spermatocytes was not associated with the XY chromosomes but was scattered throughout the nucleus. Quantitation of NEK1 fluorescence intensity staining (shown as fold change) in Setx ${ }^{+/+}$and Setx ${ }^{-/-}$. Scale bar, $20 \mu \mathrm{m}$. Dotted circle, XY chromosomes. All data were plotted as the mean \pm S.d. Statistical analysis was performed using the Student's $t$-test, ${ }^{*} P<0.05$. Number of animals, $n=3$.

spermatocytes, but rather formed a diffuse staining pattern throughout the nucleus. Quantitation of fluorescence intensity of NEK1 also showed an $\sim$ 3.6-fold reduction in $\operatorname{Set}^{-/-}$as compared with Setx ${ }^{+/+}$ (Figure 4b). The decreased protein level of NEK1 as well as its mislocalization in $\operatorname{Setx}^{-/-}$spermatocytes suggests that senataxin may modulate the expression indirectly and/or localization of NEK1, and that NEK1 disruption may in turn account for the destabilization of ATRIP and the defect in ATR signaling observed in $\operatorname{Set}^{-/-}$spermatocytes.

\section{Lack of CHD4 recruitment to the $X Y$ body in the absence of senataxin}

We have previously shown that in the absence of senataxin, failure of MSCI is associated with differential chromatin conformation as observed by defective ubiquitination of histone H2A [20]. The abnormal ATR activation and signaling observed in Setx $x^{-1-}$ spermatocytes and the alterations in $\mathrm{X}$ - and $\mathrm{Y}$-linked gene expression in $\operatorname{Set}^{-/-}$spermatocytes observed previously [20] could also result from defective chromatin remodeling. Relevant to this, the CHD4, a member of the NuRD complex [36], was shown previously to interact with senataxin in HeLa cells [16]. Gene expression connectivity mapping provided additional evidence that senataxin and CHD4 are interacting partners (Figure 5a). Connectivity mapping is an advanced bioinformatics technique that is used to establish the connections among different biological states via their expression profiles/signatures [37]. In addition to identifying CHD4 as a senataxininteracting protein, other proteins implicated in transcription control were also identified (Figure 5a). CHD4 has also been reported to interact with ATR [38], suggesting a link between its role in mediating checkpoints induced by DNA damage and chromatin remodeling by deacetylation [39]. Immunostaining for CHD4 revealed a similar pattern of localization to the $\mathrm{XY}$ chromosomes in Setx ${ }^{+/+}$and Setx ${ }^{-/-}$spermatocytes (Figure 5b). Quantitation of CHD4 fluorescence intensity staining on the $\mathrm{XY}$ chromosomes revealed an $\sim 5$-fold reduction in Setx ${ }^{-/-}$compared with $\operatorname{Set}^{+/+}$ (Figure 5c). This was not explained by reduced protein, 
a

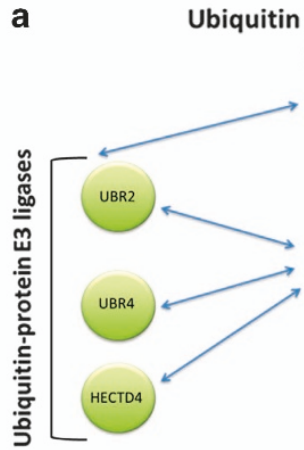

C

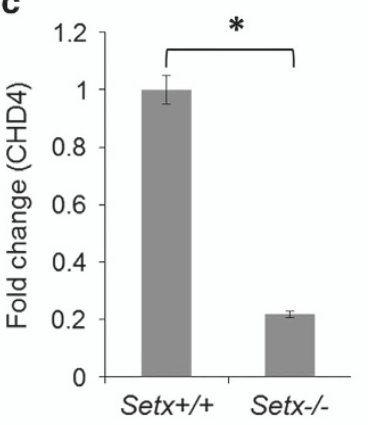

b

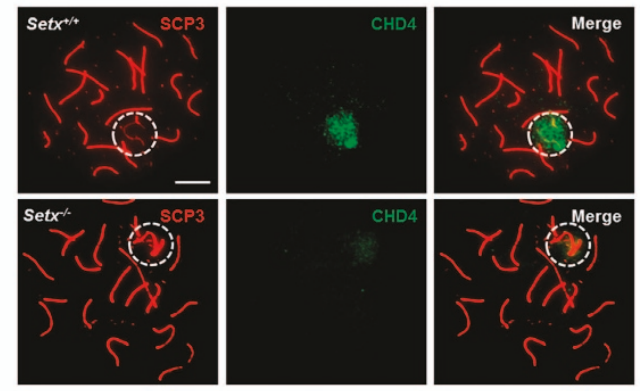

e

d
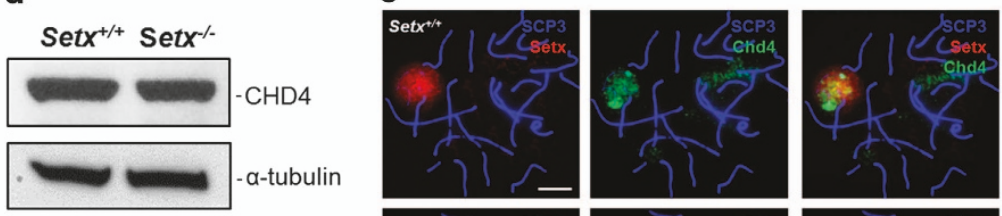

Setx
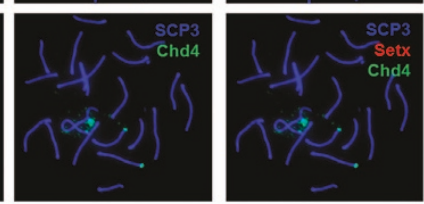

f
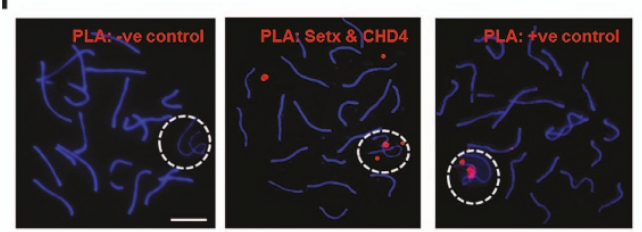

Figure 5 Senataxin interacts with and affects chromodomain helicase binding DNA protein 4 (CHD4) localization. (a) Pathway analysis of the senataxin connectivity map was performed using data from PCViz Pathway Commons Network Visualizer (http:// www.pathwaycommons.org); this demonstrated that senataxin was coregulated with three ubiquitin ligases and an ubiquitin peptidase, along with two other helicases. (b) Immunostaining for CHD4 revealed a strong signal on the XY chromosomes in Setx $^{+/+}$spermatocytes, whereas decreased levels of CHD4 were observed in Setx ${ }^{-/}$spermatocytes. (c) Quantitation of CHD4 fluorescence intensity staining (shown as fold change) in Setx ${ }^{+/+}$and Setx ${ }^{-/-}$. All data were plotted as the mean \pm s.d. Statistical analysis was performed using the Student's $t$-test, ${ }^{*} P<0.05$. Number of animals, $n=3$. (d) Immunoblotting from testes extracts for CHD4 revealed similar levels of this protein in both Setx ${ }^{-/-}$and Setx ${ }^{+/+}$mice. (e) Co-immunofluorescence of senataxin and CHD4 revealed overlapping staining over the $X Y$ domain in Setx ${ }^{+/+}$spermatocytes. (f) Proximity ligation assay (PLA) between senataxin and CHD4 revealed an interaction between these proteins at the XY chromosomes in spermatocytes of Setx ${ }^{+/+}$mice (middle panel). A negative control without primary antibody (left panel) as well as a positive control (right panel) using antibodies against breast cancer type 1 susceptibility protein (BRCA1) and ATR, two proteins known to interact directly, was performed alongside. Scale bar, $20 \mu \mathrm{m}$. Dotted circle, XY chromosomes.

as similar levels of CHD4 protein were observed in both $\operatorname{Set}^{+/+}$and $\operatorname{Set}^{-/-}$testes extracts (Figure 5d), indicating that the faint CHD4 staining in Setx $x^{--}$ spermatocytes most likely resulted from a defect in its recruitment to the XY chromosomes. Although CHD4 was shown to interact with senataxin by co-immunoprecipitation [16] and predicted from connectivity mapping in somatic cells, it was important to determine whether senataxin and CHD4 interacted in vivo in spermatocytes. Immunostaining for senataxin and CHD4 revealed colocalization on the $\mathrm{XY}$ chromosomes (Figure 5e). Using PLA, we revealed an in situ interaction between senataxin and CHD4 at the XY chromosomes in Set $^{+/+}$spermatocytes (Figure 5f), thus providing further evidence for the in vivo interaction between these two proteins. This suggests that senataxin and ATR are involved in the recruitment of CHD4 to the XY body where they function together in chromatin remodeling required for $\mathrm{XY}$ silencing. 
Persistence of histone acetylation on the $X Y$ chromosomes in Setx ${ }^{-1-}$ spermatocytes

CHD4 functions as part of the NuRD complex to control the programming of cell states during development, triggering chromatin conformation changes by posttranslational modifications, and consequently changes in transcriptional activity [38-40]. This complex is capable of both opening and closing chromatin, with a recent report demonstrating that CHD4 is the most frequent participant in chromatin closing events [41]. A defect in the recruitment of CHD4 to the XY body is predicted to result in more open chromatin, compatible with the presence of active RNA polymerase II transcription and MSCI failure in Setx $x^{-1}$ spermatocytes [20]. To address this further, we determined the status of histone posttranslational modifications such as histone H3 acetyl K9 (H3K9Ac), a histone modification associated with transcription [42]. As expected, this modification was only observed on the autosomes and not on the XY chromosomes in Set $^{+/+}$pachytene-staged spermatocytes, consistent with the onset of MSCI, whereas H3K9Ac strongly lit up the XY chromosomes in Setx ${ }^{-1-}$ spermatocytes (Figure 6a). Quantitation of H3K9Ac fluorescence intensity staining on the $\mathrm{XY}$ chromosomes revealed an $\sim 17$-fold increase in $\operatorname{Set}^{-/-}$compared with Setx $^{+/+}$ (Figure 6b). Further evidence of open chromatin on the $\mathrm{XY}$ chromosomes was provided by analyzing the spread of histone $\mathrm{H} 4$ at lysine 16 (H4K16Ac), another histone modification associated with transcriptionally active chromatin [42]. Similarly, H4K16Ac signal was not on the XY chromosomes of Set $x^{+/+}$spermatocytes, whereas a strong signal was observed on the XY chromosomes of Set $^{-1-}$ spermatocytes with additional background signals observed on the autosomes (Figure 6c). Quantitation of H4K16Ac fluorescence intensity staining on the $\mathrm{XY}$ chromosomes revealed an $\sim 5$-fold increase in $\operatorname{Setx}^{-/-}$compared with $\operatorname{Set}^{+/+}$ (Figure 6d). Contrary to acetylation, histone methylation is an indicator of transcriptional repression [42]. To confirm the major difference in chromatin conformation in Setx ${ }^{-1-}$ spermatocytes, H3K4me1, an epigenetic marker associated with transcription repression [42], was also examined. Immunostaining against this marker revealed a strong signal over the XY chromosomes in Set $x^{+/+}$spermatocytes, consistent with transcriptional repression and silencing (Figure 6e). In contrast, signal for H3K4me1 was not observed on the XY chromosomes of Setx ${ }^{-/-}$spermatocytes but enhanced signals for this marker was observed on the autosomes, suggesting a more general genome-wide defect in chromatin topology in Set $x^{-/-}$ mice (Figure 6e). Quantitation of H3K4me1 fluorescence intensity staining on the XY chromosomes revealed an $\sim 2.5$-fold reduction in Set $x^{-1-}$ compared with $\operatorname{Set}^{+/}{ }^{+}$(Figure 6f). Altogether, the pattern of histone acetylation and methylation points to abnormalities in chromatin conformation leading to gene activation on the $\mathrm{XY}$ body, which is consistent with the MSCI failure previously observed in Setx $x^{-1-}$ pachytene-staged spermatocytes.

\section{Concerted action of senataxin and CHD4 in regulating $X Y$ silencing}

Based on the localization and in situ interaction of senataxin and CHD4 on the XY chromosomes and the aberrant histone acetylation marks observed in Set $^{-1-}$ pachytene spermatocytes, we decided to investigate the relationship between R-loop formation, senataxin and CHD4 in modulating XY silencing. GC skew is a common characteristic of human $\mathrm{CpG}$ islands and $\mathrm{R}$-loops have been shown to interfere with $\mathrm{CpG}$ island methylation at promoter sites, which can then lead to silencing defects [43]. To gain further mechanistic insight into MSCI, we first determined whether R-loop formation may modulate the expression of XY genes by using microarray analysis, and subsequently carrying out in silico analysis for R-loops formation sites (RLFS) on aberrantly expressed XY genes in Setx ${ }^{-/-}$. The mouse Timpl gene, which displayed increased expression in $\operatorname{Setx}^{-/-}$(Figure 7a), was found to contain a predicted RLFS upstream of its 5'-untranslated region (UTR) (Figure $7 \mathrm{~b}$ ). We next performed DNA:RNA immunoprecipitation (DRIP) using the S9.6 antibody, an antibody specific for R-loops (Supplementary Figure S4), to confirm the presence of these structures at this locus. As shown in Figure 7c, a higher level of R-loops ( 2-fold increase) was observed at the Timpl locus in Setx $x^{-/-}$as compared with Setx ${ }^{+/+}$. Chromatin immunoprecipitation (ChIP) with senataxin also revealed its binding to this locus in Setx $x^{+/+}$. As expected, only background levels of binding were observed in Setx ${ }^{-/-}$(Figure 7c). These data are in agreement with the role of senataxin in resolving R-loops [13]. Interestingly, the 5'-UTR region of Timpl has been found to be methylated in germ cells under normal conditions (Supplementary Figure S5), suggesting that the absence of senataxin may lead to an increase in R-loop formation at this locus, which may prevent the methylation of this GC-rich region in $\operatorname{Set}^{-1-}$.

Gene regulation is primarily modulated by histone methylation and/or acetylation [44, 45]. Given the in situ interaction between senataxin and CHD4 on the 
a
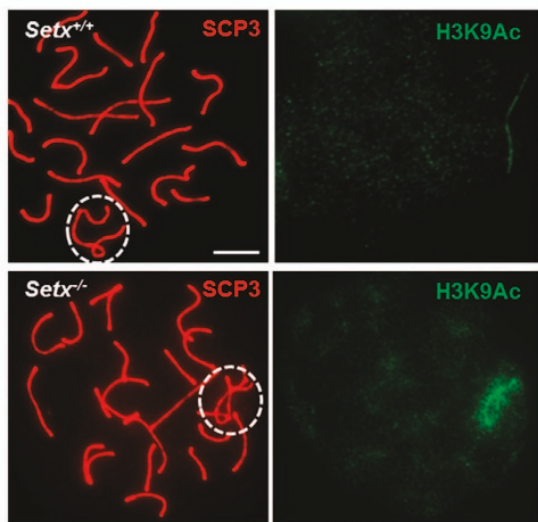

c
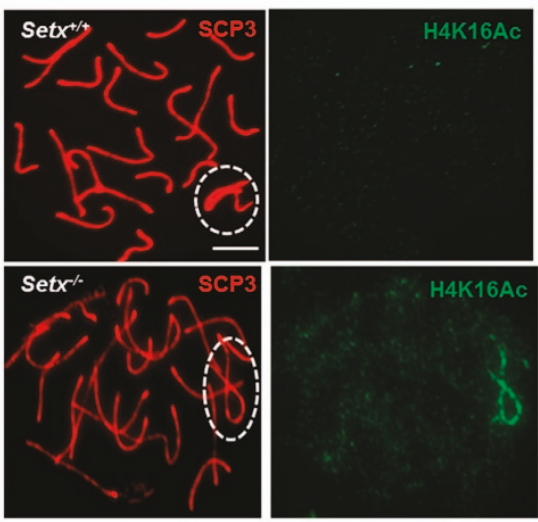

e
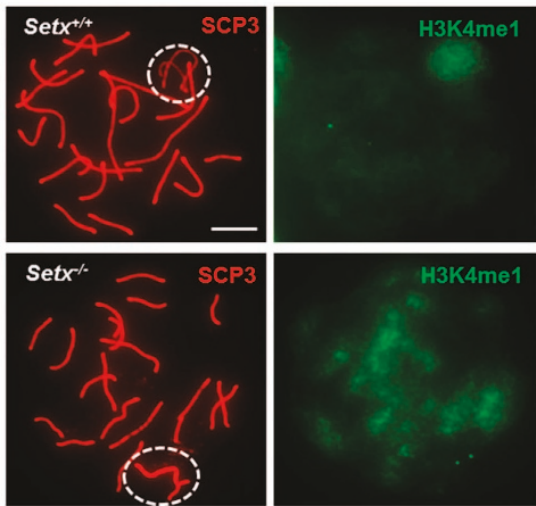
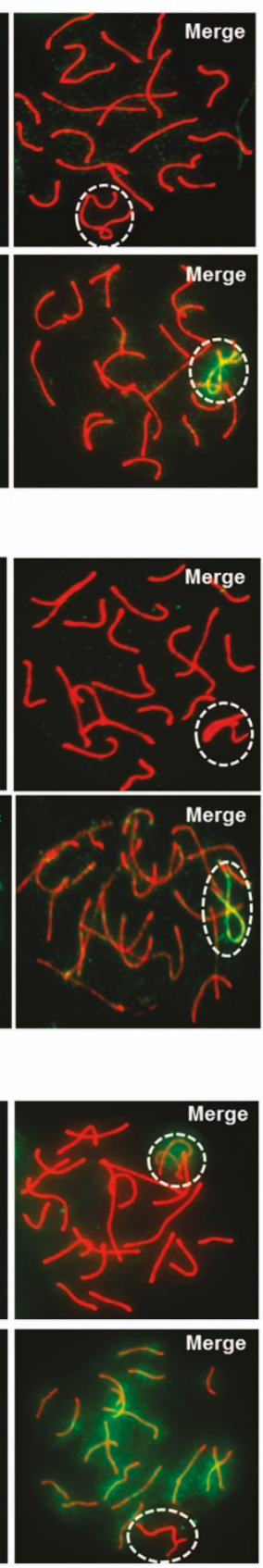

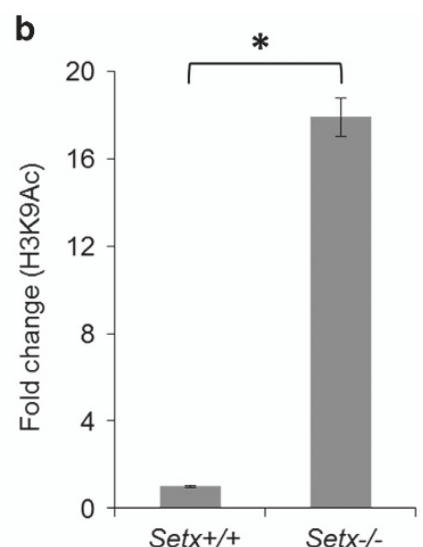

d

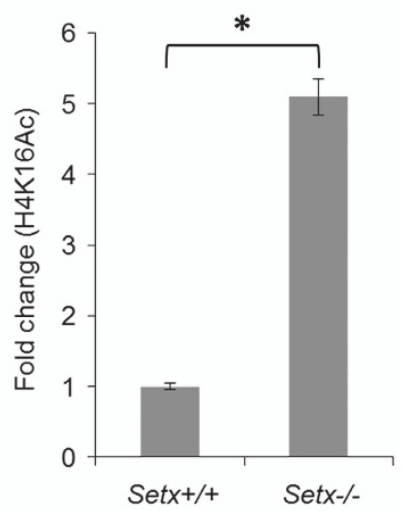

f

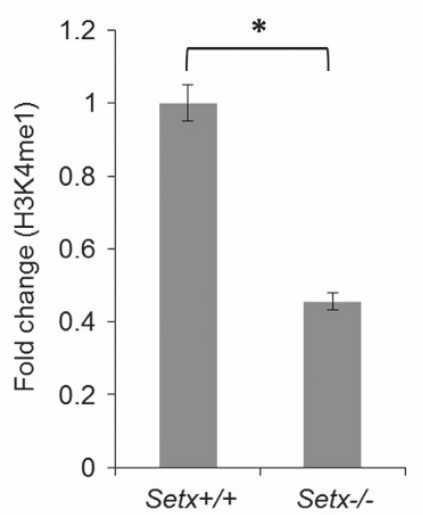

Figure 6 Differential $\mathrm{XY}$ chromatin topology contributes to the failure of meiotic sex chromosome inactivation (MSCl) in Setx ${ }^{-/-}$ spermatocytes. (a) Immunostaining for the epigenetic marker of acetylation, histone H3 acetyl K9 (H3K9Ac), revealed hyperacetylation of the $\mathrm{XY}$ chromosomes only in Setx ${ }^{-/}$spermatocytes. No signals for these histone modifications were observed over the XY domain of Setx ${ }^{+/+}$spermatocytes as expected. (b) Quantitation of H3K9Ac fluorescence intensity staining (shown as fold change) in Setx ${ }^{+/+}$and Setx ${ }^{-/-}$. (c) Histone H4 at lysine 16 (H4K16Ac) staining revealed similar hyperacetylation of the XY chromosomes only in Setx ${ }^{-/}$spermatocytes. (d) Quantitation of H4K16Ac fluorescence intensity staining (shown as fold change) in Setx ${ }^{+/+}$and Setx ${ }^{-/-}$. (e) Immunostaining for the histone methylation mark, H3K4me1, revealed specific staining over the

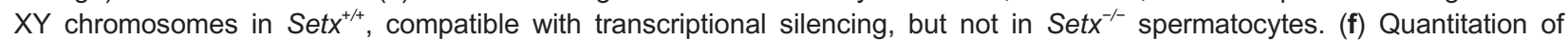
H3K4me1 fluorescence intensity staining (shown as fold change) in Setx ${ }^{+/+}$and Setx ${ }^{-/-}$. Scale bar, $20 \mu m$. Dotted circle, $X Y$ chromosomes. All data were plotted as the mean \pm s.d. Statistical analysis was performed using the Student's $t$-test, ${ }^{*} P<0.05$. Number of animals, $n=3$. 
a

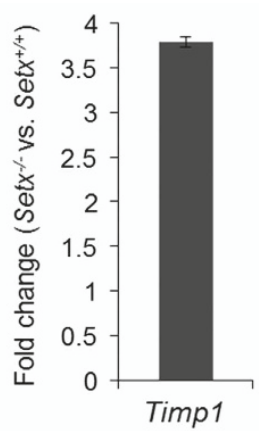

b
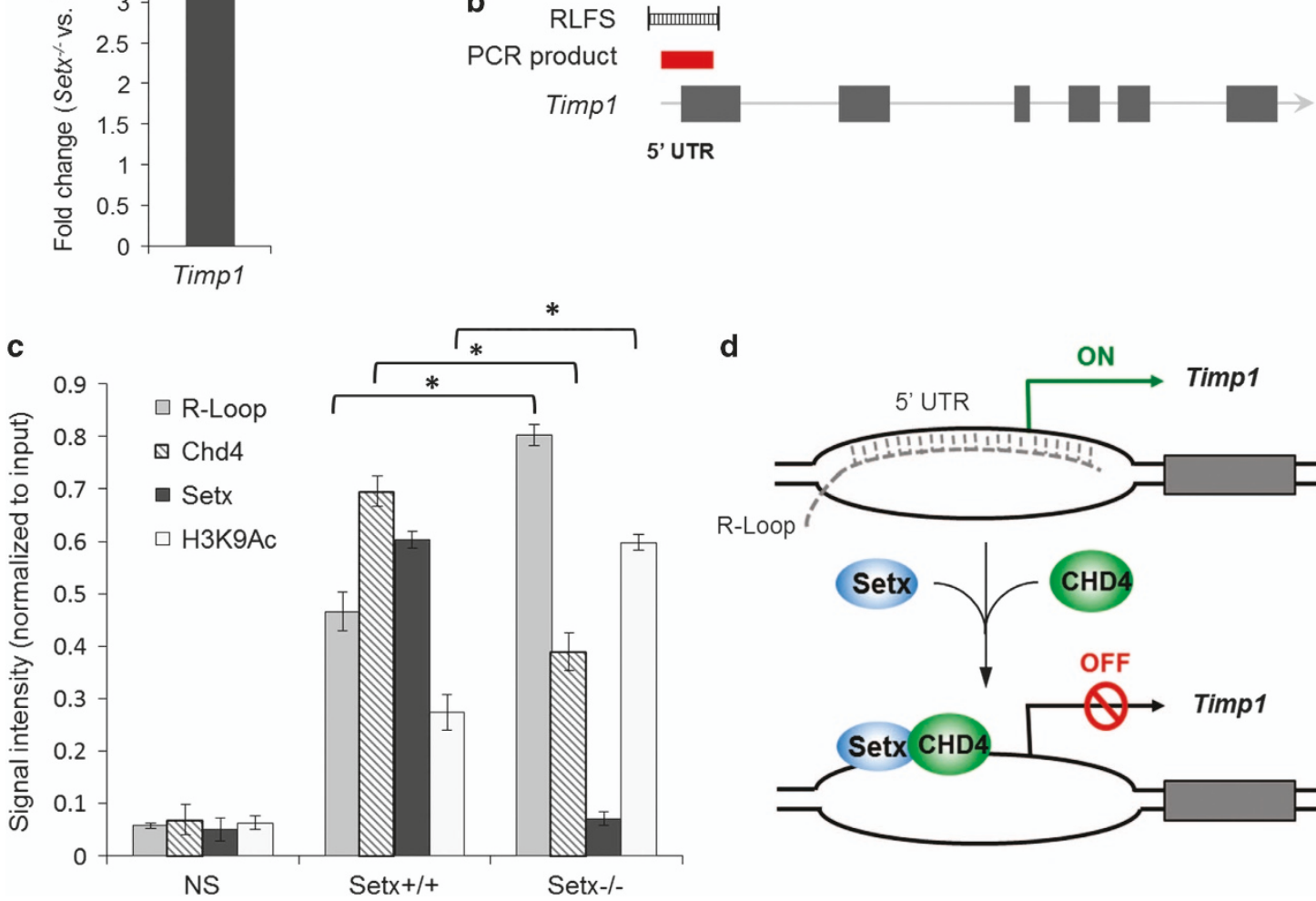

d
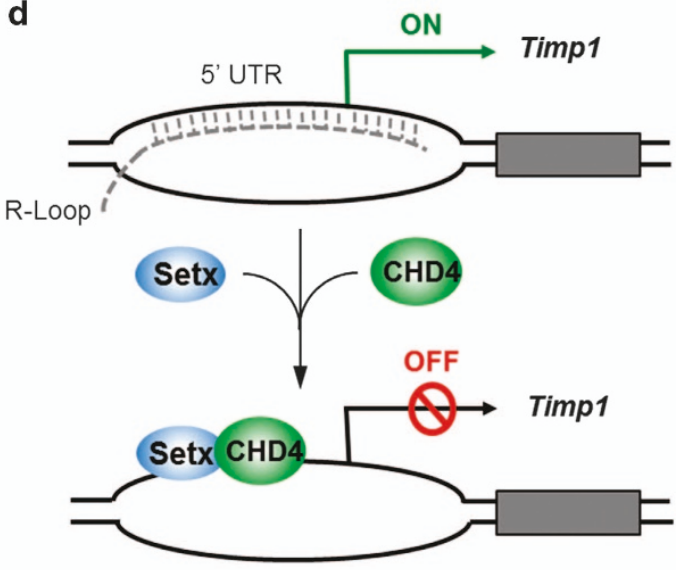

Figure 7 Coordinated action of senataxin and chromodomain helicase DNA-binding protein 4 (CHD4) in meiotic silencing. (a) Increased levels of expression of Timp1 in Setx ${ }^{-/}$germ cells. (b) Diagram depicting the Timp1 gene structure, the predicted R-loop forming site (RLFS; black bars) located upstream and in the $5^{\prime}$-untranslated region (5'-UTR) region of this gene and the corresponding polymerase chain reaction (PCR) product (red bar) used in chromatin immunoprecipitation (ChIP) assays. (c) DNA:RNA immunoprecipitation (DRIP), SETX, CHD4 and histone H3 Lys 9Ac (H3K9Ac) ChIP in Setx ${ }^{+/+}$and Setx ${ }^{-/-}$germ cells revealed the accumulation of R-loops, the reduced binding of CHD4 and the increased of histone H3 Lys9 (H3K9) acetylation in Set $x^{-/-}$cells. Similar levels of senataxin and CHD4 binding was observed in Setx ${ }^{+/+}$germ cells, whereas an $\sim 2$-fold reduction in CHD4 recruitment and only background levels of binding for senataxin similar to those obtained with negative control (nonspecific (NS)) immunoglobulin G (IgG) were observed in the knockout. All data were plotted as the mean \pm s.d. Statistical analysis was performed using the Student's $t$-test, ${ }^{*} P<0.05$. Number of animals, $n=3$. (d). Model depicting the coordinated action of senataxin and CHD4 in silencing Timp1 during meiosis. Briefly, under normal conditions R-loops form at the GC-rich region upstream at the 5'-UTR of the Timp1 gene. Recruitment of senataxin and CHD4 occurs and leads to R-loop resolution, histone deacetylation and silencing of Timp1 during meiosis.

$\mathrm{XY}$ chromosomes, and that CHD4 is a core component of the histone deacetylase NuRD complex [46], we next performed ChIP to investigate the recruitment of CHD4 to the Timp1 locus and the histone acetylation status of this region. As shown in Figure 7c, CHD4 bound to this region to a similar extent as senataxin in $\operatorname{Set}^{+/+}$, whereas an $\sim 50 \%$ reduction in CHD4 binding was observed in $\mathrm{Setx}^{-1-}$. Additionally, reduced CHD4 binding to this locus was inversely correlated with a twofold increase in histone H3 Lys9 (H3K9) acetylation, which is in agreement with previous work demonstrating a role for CHD4 in modulating the modification of this site [46].
Altogether, these data indicate that (i) R-loops form at the 5'-UTR region of the Timpl locus under normal conditions, (ii) R-loops accumulate to a greater extent at this site in the absence of senataxin and (iii) the lack of senataxin hinders the recruitment of CHD4, which correlates with higher levels of histone acetylation at this locus and consequently increased Timpl expression. Thus, we propose a mechanism in which under normal conditions, the concomitant binding of senataxin and CHD4 to Timp1 5'-UTR leads to the silencing of Timpl through the resolution of R-loops and the deacetylation of H3K9 by the CHD4 (Figure 7d). In contrast, R-loops accumulate at the 
5'-UTR in the absence of senataxin, preventing methylation of the region, and as CHD4 is not efficiently recruited, $\mathrm{H} 3 \mathrm{~K} 9$ acetylation is not removed, thus leading to the aberrant expression of Timpl during MSCI in $\operatorname{Set}^{-/-}$.

\section{Discussion}

We have previously shown that the disruption of Setx in mice leads to a defect in meiotic recombination and a failure in MSCI [20]. In this report, we have provided greater mechanistic insight into the role of senataxin in MSCI. Recent data suggest that meiotic silencing involves a complex interplay between ATR and other silencing effectors [47]. In that study, ATR was shown to have multiple roles in silencing, including the regulation of HORMA (Hop1, Rev7, Mad2) domain protein HORMAD1/2 phosphorylation and localization of ATRIP and TopBP1 to unsynapsed axes of the XY chromosomes. ATR subsequently transduces signaling at unsynapsed axes to the surrounding chromatin with the assistance of MDC1 and phosphorylates $\mathrm{H} 2 \mathrm{AX}(\gamma \mathrm{H} 2 \mathrm{AX})$, the epigenetic event leading to gene inactivation. We have previously shown that in the absence of senataxin, while ATR localizes to the axial element of XY chromosomes, it does not diffuse out into the surrounding chromatin [20]. Nevertheless, under these conditions, phosphorylation of H2AX (across the XY body) appeared to be normal, suggesting that since ATR is responsible for this phosphorylation, it was not required to localize to the surrounding chromatin to achieve this or that perhaps another PIKK was involved in this phosphorylation. Royo et al. [47] envisaged that when ATR translocates into chromatin loops of unsynapsed regions, it leads to the induction of repressive posttranslational modifications, such as $\gamma \mathrm{H} 2 \mathrm{AX}$, and causes irreversible gene silencing over several megabases. Our results suggest that phosphorylation of $\mathrm{H} 2 \mathrm{AX}$ is not the rate-determining step in gene silencing and that other modifications to chromatin are also required.

As part of the response to DNA damage, ATRIP forms a stoichiometric complex with ATR and by binding directly to RPA, enables the accumulation of ATR at the sites of DNA damage [48]. Taken together with the involvement of a number of other regulatory factors, this leads to ATR activation to coordinate cell cycle checkpoints, replication fork stability and replication restart [49]. Not surprisingly, the mechanism of ATR activation is conserved in meiosis where ATRIP localizes with ATR, TopBP1 and RPA at unsynapsed regions of meiotic chromosomes [50]. A recent report revealed that ATRIP is SUMOylated in response to agents that disrupt DNA replication and that ATRIP mutants lacking SUMOylation sites failed to localize to DNA damage sites and did not support efficient ATR activation [51, 52]. In promoting ATR activation, SUMOylated ATRIP interacts with multiple proteins including ATR, TopBP1, RPA and the MRN complex not only through direct interaction but also through interaction with SUMO proteins. Here we revealed a marked reduction in SUMOylated proteins that localize to the XY body in $\operatorname{Set}^{-/-}$spermatocytes and that senataxin, which localizes preferentially to the XY body during the pachytene stage, is also SUMOylated. It is possible that SUMOylated senataxin has a role in DDR protein-mediated MSCI. Indeed, we showed that ATRIP localized predominantly to the axial elements of the XY chromosomes but when senataxin was disrupted there was a significant reduction of its association with the XY chromosomes. This might be explained by lack of SUMOylation. It is also evident from immunoblotting that in the absence of senataxin, the protein levels of ATRIP present was markedly reduced, providing an explanation for the reduced ATRIP SUMOylation observed in Setx ${ }^{-/-}$spermatocytes. The lower levels of ATRIP and its markedly reduced recruitment to the axial elements of $\mathrm{XY}$ chromosomes are likely to contribute to the abnormality in ATR activity. Our data suggest that decreased levels of NEK1 and its mislocalization in Set $x^{-/}$ pachytene cells accounts for the reduced levels of ATRIP. Liu et al. [35] showed that even in undamaged cells, NEK1 is required for maintaining levels of ATRIP, the association between ATR and ATRIP, and the basal kinase activity of ATR [35]. In this way, NEK1 primes the ATR-ATRIP heterodimer for a robust DNA damage response. Thus, the defect in NEK 1 and the consequent reduction in ATRIP protein levels observed in Setx ${ }^{-1-}$ pachytene spermatocytes could account for the defective activation and signaling through ATR leading to the failure of MSCI.

The great majority of SUMOylation staining was localized to the XY body and in the absence of senataxin, this is markedly reduced. As discussed above, ATRIP was one of several SUMOylated proteins associated with the XY body during meiosis. Others include ATRX, which is a chromatin remodeling protein shown recently to promote the incorporation of histone variant $\mathrm{H} 3.3$ at particular transcribed genes to facilitate transcriptional elongation through G-rich sequences [53]. This is of interest as we showed that senataxin is also SUMOylated and localizes to the XY 
body. The capacity of senataxin to resolve R-loops $[13,14,19,20]$ and the propensity of GC-rich repeats to form not only G-quadruplexes but also R-loops, leading to abortive transcription [54], supports a common goal for these two proteins in XY inactivation, ensuring that transcription is completed on the $\mathrm{XY}$ chromosomes before entry into the diplotene stage. Other SUMOylated proteins include DHX15, a DEAH-box helicase that is part of the splicesosome complex and contributes to the activation of signaling pathways [55]; RUVB-like ATPase that functions in chromatin decondensation [56] and YBOX transcription factors. Clearly, all of these proteins have the potential to be involved in remodeling chromatin on the XY chromosomes to ensure the onset of gene silencing or they could contribute to the re-emergence of transcriptional activation at later stage of meiosis.

The marked deficiency in the recruitment of CHD4 to the XY body in $\operatorname{Set}^{-l-}$ pachytene cells and its role in transcriptional regulation substantiates further a central role for senataxin in gene silencing. CHD4 is a core component of the NuRD corepressor complex that remodels chromatin in a conformation refractory to active transcription $[57,58]$. We provided evidence for the first time that CHD4 interacts with senataxin in situ on the XY body, which confirms the interaction between the two proteins demonstrated in proliferating cells using affinity chromatography, mass spectrometry and immunoblotting [16]. CHD4 has also been shown to interact with ATR [39]. In that study, ATR was shown to be in a complex that included CHD4, HDAC2 and other members of the NuRD complex, suggesting that as part of the complex ATR would have greater access to damaged DNA. CHD4 was subsequently found to localize to sites of DNA damage and cells depleted of this protein were hypersensitive to DNA damaging agents, exhibited cell cycle abnormalities and retained DNA DSBs for extended periods of time [59]. It was suggested that in CHD4-deficient cells, either the access of DNA repair proteins to the site of damage is compromised or that the deficiency of CHD4 leads to global alterations in higher-order chromatin structure, rendering it more vulnerable to accumulating DNA damage. Our data indicate that CHD4 plays a similar role in remodeling chromatin to allow access of silencing factors to asynapsed regions of the XY chromosomes to turn off or prevent transcription during meiosis. The defective recruitment of CHD4 to the XY body in Setx ${ }^{-1-}$ pachytene cells is expected to result in more open chromatin since it normally functions as a repressor of transcription $[37,46]$. CHD4, a part of the NuRD histone deacetylase complex, is also involved in removing acetylation marks from histones to convert open transcriptionally active euchromatin into closed transcriptionally inactive heterochromatin. Indeed, higher levels of acetylated histones such as H3K9Ac and H4K16Ac were observed on the XY body in Setx ${ }^{-1-}$. Although H3K9Ac is present at low levels in the early stages of prophase 1 , it increases strongly by midpachytene on autosomes but is excluded from XY chromosomes which are initiating MSCI [42]. H3K9Ac strongly decorated the XY body in $\operatorname{Set}^{-/-}$cells, which is consistent with a failure of transcription repression in Setx $^{-l-}$ pachytene cells. Furthermore, H3K4me1, a marker of transcriptionally inactive heterochromatin, was excluded from the XY body in Setx ${ }^{-/-}$spermatocytes, demonstrating the important role of senataxin in coordinating CHD4 recruitment, histone modification and transcriptional silencing. This is in agreement with the MSCI failure and abnormal expression of $\mathrm{X}$ - and Y-linked genes observed previously [20]. Although H3K4mel has generally been associated with transcriptional activation, several reports have been published discussing its role in silencing. Page et al. [42] showed H3K4mel localization to the XY body during the pachytene stage, which prompted the authors to suggest its involvement in transcriptional silencing during MSCI. Additionally, Cheng et al. [60] described its association with transcriptional silencing in embryonic fibroblasts, macrophages and human embryonic stem cells. Thus, there exists evidence suggesting both a role in transcriptional activation and silencing for $\mathrm{H} 3 \mathrm{~K} 4 \mathrm{me} 1$, which may depend on the cell type investigated.

We have provided important mechanistic insight into gene silencing of the XY chromosomes in meiosis and the central role of senataxin in this process, involving activation of ATR, recruitment of the chromatin remodeler CHD4 and the ensuing posttranslational histone modifications necessary for MSCI. These new data provided additional insight into the sequence of events involved in MSCI (Figure 8) at the pachytene stage. The initial step is BRCA1dependent recruitment of ATR and that of senataxin as we have recently demonstrated [20]. In the absence of senataxin, ATR, ATRIP and TopBP1 are all retained on the axial element of the XY chromosomes. Additionally, the protein levels of both ATRIP and TopBP1 are reduced as well as their binding to the XY chromosomes. Under these conditions, we did not detect diffusion of ATR or its downstream substrates, although normal levels of $\mathrm{H} 2 \mathrm{AX}$ phosphorylation were detected over the XY body. This suggests that another 

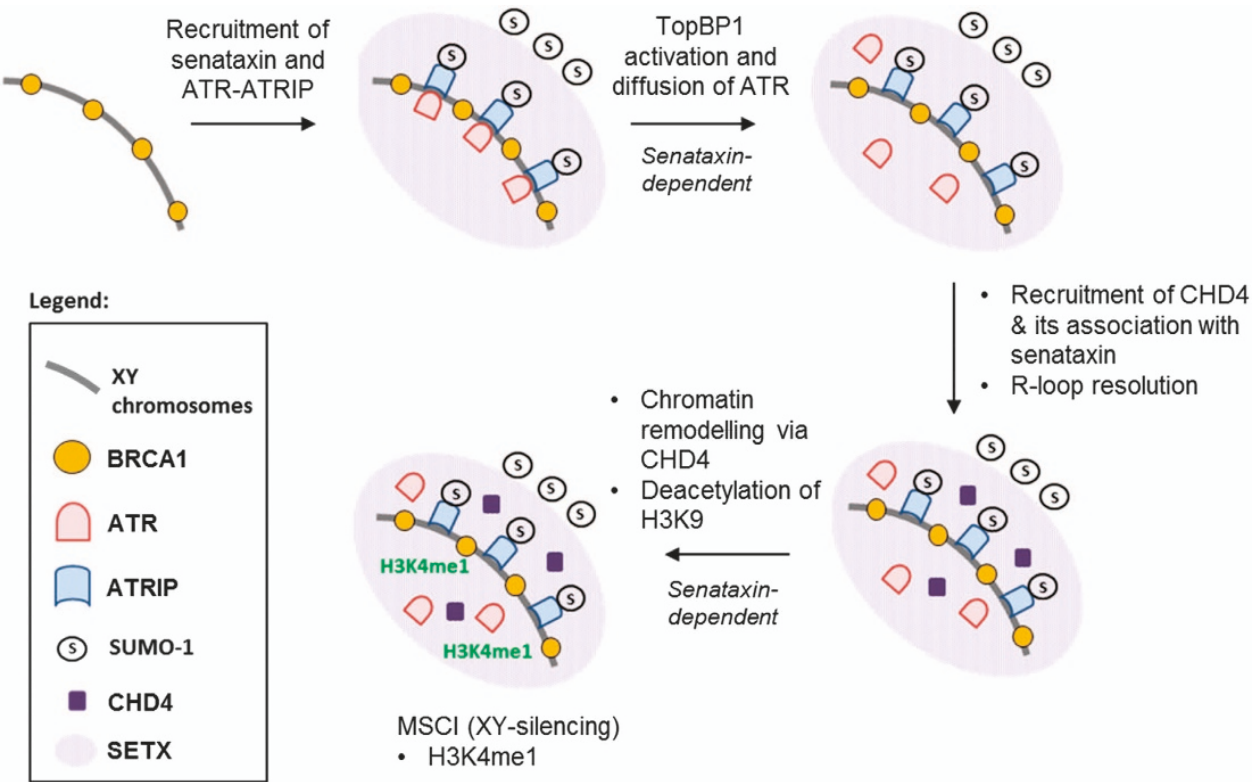

- H3K4me1

Figure 8 Integrated role of senataxin in meiotic sex chromosome inactivation (MSCI). MSCl is initiated with breast cancer type 1 susceptibility protein (BRCA1) first coating the axial element of the XY chromosomes during the pachytene stage. This is followed by recruitment of senataxin and ataxia telangiectasia and Rad3-related protein (ATR)-interacting partner (ATR/ATRIP) where both senataxin and ATRIP are present as SUMOylated proteins. Binding of TopBP1 activates ATR, which then disperses into the $X Y$ chromatin domain where it functions to phosphorylate other proteins, which may include histone $\mathrm{H} 2 \mathrm{AX}(\gamma \mathrm{H} 2 \mathrm{AX})$. This step is senataxin-dependent. ATR diffusion into surrounding chromatin is not essential for H2AX phosphorylation as this occurs in the absence of senataxin where ATR is confined to the axial elements of XY chromosomes. Senataxin acts to resolve R-loops on XY chromosomes and associates with CHD4, which is part of the nucleosome remodeling complex (NuRD), to assist in the deacetylation of histones to downregulate active gene expression. The remodeling of chromatin for $\mathrm{MSCl}$ is dependent on senataxin. In addition, monomethylation of H3K4 occurs, all of which gives rise to heterochromatin formation and $\mathrm{XY}$ silencing.

PIKK distinct from ATR can carry out this phosphorylation. Although $\gamma \mathrm{H} 2 \mathrm{AX}$ is present, it does not ensure that MSCI will occur, suggesting that it is not a single determinant but is instead only one of several required chromatin modifications to achieve gene silencing. We provided further insight into this by demonstrating the requirement for senataxin to recruit CHD4 to the XY chromosomes and that these two proteins interact at the level of the XY body. Specific evidence of a concerted action between senataxin and CHD4 on the XY chromosomes was demonstrated at the X-linked Timpl gene (Figure 7d). The higher levels of histone acetylation modifications, which were observed in $\operatorname{Setx}^{-/-}$spermatocytes, have been previously associated with aberrant active gene transcription for $\mathrm{X}$ - and $\mathrm{Y}$-linked genes during pachytene [20]. This is also in agreement with increased levels of R-loop formation observed in Setx $x^{-/-}$ spermatocytes, which may also contribute to the failure of MSCI and inhibition of meiosis [19, 20]. In summary, senataxin has been demonstrated to facilitate transcription termination by resolving R-loops [13], coordinate transcription with meiotic recombination
[20], act at the interface of transcription, DNA damage response and RNA surveillance [5, 16] and protect against DNA DSBs generated in transcription-coupled DNA repair [61]. The findings in this report adds a new level of complexity to senataxin's function in meiosis by demonstrating a direct role in modulating chromatin remodeling and epigenetic gene silencing via CHD4 and the ATR signaling pathway, and highlights its pivotal role in coordinating gene regulation and the DNA damage response.

\section{Materials and Methods}

\section{Animal husbandry and genotyping}

All animal experiments were approved by the QIMR Berghofer Medical Research Institute Animal Ethics Committee, The University of Queensland. The mice were weaned at 21 days postpartum and ear clipped for identification. Genotyping was carried out via polymerase chain reaction (PCR) on genomic DNA isolated from tail tips as described in Becherel et al. [20].

\section{Spermatocyte spreads}

All spreads were made from testes collected from adult 35-day-old mice. Briefly, testes were decapsulated, finely 
chopped and rinsed in $1 \mathrm{ml}$ of GIBCO serum-free DME medium (Life Technologies, Carlsbad, CA, USA) containing $1 \times$ Complete Protease Inhibitor (Roche, Basel, Switzerland). Six microliters of GIBCO serum-free DME medium (Life Technologies) was then added and tubes were allowed to stand on ice for $5 \mathrm{~min}$ to allow large clumps and cellular debris to settle. Six microliters of the remaining supernatant from each sample was subsequently aliquoted into Eppendorf tubes, with each tube containing $1 \mathrm{ml}$ of suspension. Tubes were then centrifuged for $5 \mathrm{~min}$ at $5000 \mathrm{~g}$ to pellet the cell suspension. The pellet was then resuspended in $0.1 \mathrm{~m}$ sucrose and applied onto glass slides prewetted with $1 \%$ paraformaldehyde and $0.1 \%$ Triton X-100 in phosphate-buffered saline (PBS). Cells were fixed on the glass slides and air-dried for $3 \mathrm{~h}$ at room temperature. The slides were subsequently washed with 1:250 Kodak Photo-Flo 200 (Kodak Professional, Rochester, NY, USA) in $1 \times$ PBS and air-dried for $1 \mathrm{~h}$. Once dried, spreads were stored at $-80{ }^{\circ} \mathrm{C}$.

\section{Immunofluorescence}

Spermatocyte spreads were blocked in $20 \%$ fetal calf serum, $2 \%$ bovine serum albumin (BSA), $0.2 \%$ Triton $\mathrm{X}-100$ in $1 \times \mathrm{PBS}$ for $1 \mathrm{~h}$ at room temperature. Slides were incubated overnight at $4{ }^{\circ} \mathrm{C}$ in a humidified chamber with the following primary antibodies diluted in blocking buffer: mouse monoclonal anti-SCP3 (Abcam, Cambridge, MA, USA; AB97672) at a 1:100 dilution, rabbit anti-SCP3 (Novus Biologicals, Littleton, CO, USA; NB300-230) at a 1:100 dilution, goat anti-ATR (Santa Cruz Biotechnology, Dallas, TX, USA; SC-1887) at a 1:100 dilution, rabbit anti-NEK1 (Bioss Inc., Woburn, MA, USA; BS-7814R) at a 1:100 dilution, rabbit anti-ATRIP (Thermo Scientific, Waltham, MA, USA; PA1-519) at a 1:100 dilution, mouse monoclonal anti-CHK1 (Cell Signaling Technology, Danvers, MA, USA; 2 360) at a 1:100 dilution, rabbit anti-CHD4 (Genetex, Irvine, CA, USA; GTX124186), sheep anti-Setx (in-house) at a 1:300 dilution, rabbit anti-TopBP1 (Abcam; AB2402) at a 1:100 dilution, rabbit anti SUMO-1 (Abcam; AB32058) at a 1:100 dilution, rabbit anti-SUMO-2/3 (Abcam; AB3742) at a 1:100 dilution, rabbit anti-phospho ATR (S428) (Cell Signaling Technology; 2853) at a 1:100 dilution, rabbit anti-phospho RPA (S33) (Bethyl Laboratories Inc., Montgomery, TX, USA; A300-246A) at a 1:100 dilution, rabbit anti-phospho CHK1 (S317) (Cell Signaling Technology; 2 344) at a 1:100 dilution, rabbit anti-H3K4me1 (Abcam; AB8895) at a 1:100 dilution, rabbit anti-H3K9Ac (Bioss Inc.; BS-3776A) at a 1:100 dilution, and rabbit anti-H4K12Ac (Bioss Inc.; BS-3746R) at a 1:100 dilution. Slides were then rinsed three times with $1 \times$ PBS containing $0.5 \%$ Triton $\mathrm{X}-100$ for $5 \mathrm{~min}$ each at room temperature on an orbital shaker. Slides were then incubated for $1 \mathrm{~h}$ at $37^{\circ} \mathrm{C}$ in a humidified chamber with the following Alexa-Dyeconjugated secondary antibodies (Life Technologies) diluted 1:250 in blocking buffer: Alexa 488 chicken anti-mouse (A-21200), Alexa 594 donkey anti-rabbit (A-11032), Alexa 594 donkey anti-goat (A-11058) and Alexa 350 goat anti-mouse (A-11045). Subsequently, slides were washed three times as before and Hoechst 33342 (1:10 000) (Life Technologies) was applied for $10 \mathrm{~min}$ to stain nuclei. Slides were finally washed two times and glass coverslips were mounted for imaging. Images were captured using a digital camera (AxioCam Mrm; Carl Zeiss Microimaging Inc., Jena, Germany) attached to a fluorescent microscope (Axioskop 2 mot plus; Carl Zeiss Microimaging) equipped with the appropriate filters, and the AxioVision 4.8 software (Carl Zeiss Microimaging Inc.). The objective used was an EC Plan-Neofluar 10x/0.3 (Carl Zeiss Microimaging Inc.). Images were subsequently assembled in Adobe Photoshop 5 (Adobe Systems, San Jose, CA, USA), and contrast and brightness were adjusted on the whole image panel at the same time. Fluorescence intensity was quantitated on at least 50 individual cells per marker and condition using the RAW images and NIH ImageJ software version 1.47 (NIH, Bethesda, MD, USA). Statistical analysis was carried out using Student's $t$-test.

\section{Proximity ligation assay}

PLA (Duolink; Olink Bioscience, Uppsala, Sweden) was performed according to the manufacturer's protocol with the following primary antibodies: mouse monoclonal anti-SCP3 (Abcam; AB97672) at a 1:100 dilution, rabbit anti SUMO-1 (Abcam; AB32058) at a 1:100 dilution, rabbit anti-phospho ATR (S428) (Cell Signaling Technology; 2853) at a 1:100 dilution, sheep anti-Setx (in-house) at a 1:300 dilution, rabbit anti-CHD4 (Abcam; AB72418), and rabbit anti-BRCA1 (with courtesy of Prof. David Livingstone, Dana-Farber Cancer Institute, Harvard Medical School, Boston, MA, USA) at a 1:300 dilution. Corresponding PLA PLUS and MINUS probes were subsequently applied. Slide mounting and imaging was performed as described above.

\section{Immunoprecipitation of SUMOylated proteins from Set $x^{+/+}$and Set $x^{-1-}$ testes}

Testes of 35-day-old mice (ground with a pestle to disrupt their structure) were lysed for $1 \mathrm{~h}$ at $4{ }^{\circ} \mathrm{C}$ on a rotating wheel with $1 \mathrm{ml}$ of lysis buffer (50 mm Tris- $\mathrm{HCl}, \mathrm{pH} 7.4,150 \mathrm{~mm} \mathrm{NaCl}$, 2 mм EGTA, 2 mм EDTA, 25 mm $\beta$-glycerophosphate, $0.1 \mathrm{~mm}$ $\mathrm{Na}_{3} \mathrm{VO}_{4}, 0.1 \mathrm{~mm}$ PMSF, $25 \mathrm{~mm} \mathrm{NaF}, 0.2 \%$ Triton $\mathrm{X}-100,0.3 \%$ NP-40 and $1 \times$ EDTA-free Complete Protease Inhibitor (Roche) $25 \mu \mathrm{M}$ NEM (Sigma-Aldrich, St Louis, MO, USA)). Cellular debris was pelleted by centrifugation at $16100 \mathrm{~g}$ at $4{ }^{\circ} \mathrm{C}$ for $10 \mathrm{~min}$, and protein concentration was determined using Lowry Assay (Bio-Rad Laboratories Inc., Hercules, CA, USA). Three micrograms of total cell extract were precleared with $20 \mu \mathrm{l}$ of protein A beads (50:50 slurry) (Merck Millipore, Billerica, MA, USA) for $3 \mathrm{~h}$ at $4{ }^{\circ} \mathrm{C}$ on a rotating wheel. Extracts were centrifuged for $5 \mathrm{~min}$ at $2000 \mathrm{~g}$, beads were removed and $10 \mu \mathrm{g}$ of anti-SUMO antibody (Abcam) was added to the extract. Extracts and antibody were incubated overnight at $4{ }^{\circ} \mathrm{C}$ on a rotating wheel to allow binding of the antibody to SUMOylated protein. The next day, $40 \mu \mathrm{l}$ of protein A beads (50:50 slurry) (Merck Millipore) was added to the extract and incubated for $2 \mathrm{~h}$ at $4{ }^{\circ} \mathrm{C}$ on a rotating wheel. Extracts were centrifuged for $5 \mathrm{~min}$ at $2000 \mathrm{~g}$ and the beads were removed. The immunoprecipitate was subsequently washed three times with lysis buffer and the beads were resuspended in SDS loading buffer containing $2 \times$ DTT and separated via $4-12 \%$ SDS-PAGE using Novex Tris-Glycine gels (Life Technologies) at $110 \mathrm{~V}$ for $1.5 \mathrm{~h}$. Once separated, gel was stained with G-250 Colloidal 
Coomassie (Life Technologies) overnight. Gel was subsequently destained using Milli-Q water.

\section{Tryptic digestion of SUMOylated proteins from mice spermatocytes for LC-MS/MS}

Bands were excised from gel using a sterile scalpel and were left to destain (50\% acetonitrile, 50\% ammonium bicarbonate) for $4 \mathrm{~h}$. Trypsin (Promega, Madison, WI, USA) was added 1:20 by weight in $50 \mathrm{~mm}$ ammonium bicarbonate until gel pieces were covered and left to digest overnight. Subsequently, 100\% acetonitrile was added and tube was vortexed for $10 \mathrm{~min}$. Liquid in the tube was then transferred into a fresh Eppendorf tube. Fifty percent acetonitrile was added to the gel pieces and vortexed again for $10 \mathrm{~min}$. Liquid was combined with the liquid collected in the previous step. Tubes were spun in a Speedy-Vac for $30 \mathrm{~min}$ to dry liquid to $\sim 5 \mu \mathrm{l}$. This volume was reconstituted to $20 \mu 10.1 \%$ trifluoroacetic acid (TFA) and applied to a C18 STAGE tip (Thermo Fisher Scientific, Waltham, MA, USA) by pipetting up and down for $\sim 10$ times. Tips were then washed with $10 \mu 1$ of $0.1 \%$ TFA and peptides were eluted with $50 \%$ acetonitrile intro a fresh Eppendorf tube. Eluant was frozen at $-20^{\circ} \mathrm{C}$ and shipped to Dr Mark E Graham (Children's Medical Research Institute, University of Sydney, NSW, Australia) for liquid chromatography mass spectrometry analyses.

\section{Liquid chromatography mass spectrometry identification of proteins immunoprecipitated from mice spermatocytes}

Coomassie-stained protein bands from the anti-SUMO-1 immunoprecipitation SDS-PAGE gel were excised using a sterile scalpel and were destained (50\% acetonitrile, 50\% ammonium bicarbonate) for $4 \mathrm{~h}$. Trypsin (Promega) was added 1:20 protein:trypsin by weight in $50 \mathrm{~mm}$ ammonium bicarbonate until gel pieces were covered and digested for $16 \mathrm{~h}$. To extract the tryptic peptides, the volume was increased by $50 \%$ with acetonitrile, the tube was vortexed for $10 \mathrm{~min}$ and the liquid was then transferred into a fresh tube. An additional solution of $50 \%$ acetonitrile in water was added to the gel pieces, the tube was vortexed for $10 \mathrm{~min}$ and the liquid was combined with the liquid from the previous step. Tubes were spun in a Speedy-Vac for $30 \mathrm{~min}$ to dry liquid to $\sim 5 \mu \mathrm{l}$. This volume was diluted to $20 \mu \mathrm{l}$ $0.1 \%$ TFA and applied to a C18 STAGE tip (Thermo Fisher Scientific) by pipetting up and down for $\sim 10$ times. Tips were then washed with $10 \mu \mathrm{l}$ of $0.1 \%$ TFA and peptides were eluted with $50 \%$ acetonitrile into a tube for storage at $-20^{\circ} \mathrm{C}$. One-half of each sample was analyzed by LC-MS/MS. A Dionex 3000 Ultimate HPLC system was used (Dionex/Thermo Fisher Scientific, Scoresby, VIC, Australia). Samples were loaded onto a trap column $(300 \mu \mathrm{m}$ inside diameter, $1 \mathrm{~mm}$ long; Acclaim PepMap100 C18, $5 \mu \mathrm{m}$, Thermo Fisher Scientific) for $5 \mathrm{~min}$ at $5 \mu 1 \mathrm{~min}^{-1}$ with phase A ( $0.1 \%$ formic acid in water). The sample was eluted through a $16 \mathrm{~cm}$ long, $75 \mu \mathrm{m}$ inside diameter analytical column $(3 \mu \mathrm{m} 120 \AA \AA$ Reprosil Pur C18-AQ; Dr Maisch Gmbh, Ammerbuch-Entringen, Germany) at $250 \mathrm{nl} \mathrm{min}^{-1}$. The gradient was from 0 to $35 \%$ phase B $(90 \%$ acetonitrile, $9.9 \%$ water and $0.1 \%$ formic acid) in $65 \mathrm{~min}$. Phase B was increased to $100 \%$ in $5 \mathrm{~min}$ and held at $100 \%$ for $5 \mathrm{~min}$. The column eluate was sprayed into an LTQ Velos orbitrap mass spectrometer (Thermo Fisher Scientific) through a $10 \mu \mathrm{m}$ inside diameter
SilicaTip (New Objective) with $2.2 \mathrm{kV}$ applied. Up to seven of the most intense peptide precursor ions above a threshold of 5000 counts were selected in the range $m / z 300-1500$ for MS/ MS for 90 min after the start of the gradient. Both MS and MS/ MS detection was in the orbitrap at a resolution of 30000 and 7500 , respectively. Detailed parameters were as follows: isolation width was $2(\mathrm{~m} / \mathrm{z})$, normalized collision energy was 40 , activation time was $0.1 \mathrm{~ms},+1$ charge state ions were rejected, ions within $100 \mathrm{ppm}$ of previously selected ions were excluded from MS/MS for $10 \mathrm{~s}$, MS target was 1000000 counts for up to $50 \mathrm{~ms}$ and MS/MS target was 50000 for up to $300 \mathrm{~ms}$. All MS raw data was submitted via Proteome Discoverer 1.3 (Thermo Fisher Scientific) for database searching with Mascot 2.4 (Matrix Science, London, UK). The database was UniProtKB (downloaded July 2013, Mus musculus, 16673 entries). Precursor ion tolerance was 10 p.p.m. and fragment ion tolerance was $0.05 \mathrm{Da}$. Variable modifications were deamidation (NQ) and oxidation (M) and maximum missed trypsin cleavages was 3 (trypsin cleavage before proline was allowed). The Mascot result files were imported to Scaffold 4.4.1 (Proteome Software, OR, USA). Only proteins with at least two unique peptides were considered. Protein false discovery rate was $0.1 \%$ and peptide false discovery rate was $0.02 \%$. Minimum protein probability of identification was $99 \%(P<0.01)$ and minimum peptide probability of identification was $95 \%(P<0.05)$.

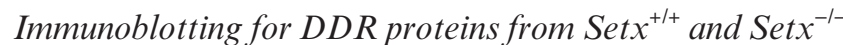 testes}

Testes of 35-day-old mice (ground with a pestle to disrupt their structure) were lysed for $1 \mathrm{~h}$ at $4{ }^{\circ} \mathrm{C}$ on a rotating wheel with $1 \mathrm{ml}$ of lysis buffer (50 mм Tris- $\mathrm{HCl}, \mathrm{pH} 7.4,150 \mathrm{~mm} \mathrm{NaCl}$, 2 mм EGTA, 2 mм EDTA, $25 \mathrm{~mm} \beta$-glycerophosphate, $0.1 \mathrm{~mm}$ $\mathrm{Na}_{3} \mathrm{VO}_{4}, 0.1 \mathrm{~mm}$ PMSF, $25 \mathrm{~mm} \mathrm{NaF}, 0.2 \%$ Triton X-100, 0.3\% NP-40 and $1 \times$ EDTA-free Complete Protease Inhibitor (Roche)). Cellular debris was pelleted by centrifugation at $16100 \mathrm{~g}$ at $4{ }^{\circ} \mathrm{C}$ for $10 \mathrm{~min}$, and protein concentration was determined using Lowry Assay (Bio-Rad). Thirty micrograms of total cell extract were resuspended in SDS loading buffer containing $2 \times$ DTT and separated via 4-12\% SDS-PAGE using Novex Tris-Glycine gels (Life Technologies) at $110 \mathrm{~V}$ for $1.5 \mathrm{~h}$. The proteins were then transferred onto a Hybond-C nitrocellulose membrane (Pall Life Sciences, NY, USA) using a highmolecular-weight transfer buffer $(100 \mathrm{~mm}$ Tris, $40 \mathrm{~mm}$ glycine, $0.05 \% \mathrm{SDS}, 20 \%$ methanol) at $100 \mathrm{~V}$ for $1 \mathrm{~h}$ at $4{ }^{\circ} \mathrm{C}$ in a miniprotean gel rig (Bio-Rad). Membranes were the blocked in 5\% skim milk in TBS/Tween-20 or 5\% BSA/TBS/Tween-20 for $1 \mathrm{~h}$ and immunoblotting was performed with the following primary antibodies diluted 1:1 000 in 5\% skim milk/TBS/Tween-20 or 5\% BSA/TBS/Tween-20 at $4{ }^{\circ} \mathrm{C}$ overnight: goat anti-ATR (Santa Cruz Biotechnology; SC-1887), rabbit anti-NEK1 (Bioss Inc., BS-7814R), rabbit anti-ATRIP (Thermo Scientific; PA1-519), mouse monoclonal anti-CHK1 (Cell Signaling Technology; 2360), rabbit anti-CHD4 (Abcam; AB72418), sheep anti-Setx (in-house), rabbit anti-TopBP1 (Abcam; AB2402), rabbit anti SUMO-1 (Abcam; AB32058), rabbit anti-phospho ATR (S428) (Cell Signaling Technology; 2853), rabbit anti-glyceraldehyde 3-phosphate dehydrogenase (Genetex; GTX100118) and rabbit anti- $\alpha$ tubulin (Genetex; GTX 112141). Membranes were 
subsequently washed thee times with TBS/Tween-20 for 5 min each and incubated with the following secondary antibodies conjugated with horseradish peroxidase (HRP) diluted 1:5000 in $5 \%$ skim milk/TBS/Tween-20 or $5 \%$ BSA/TBS/Tween- 20 for $1 \mathrm{~h}$ at room temperature: HRP-conjugated donkey anti-sheep (Merck Millipore; AP184P), HRP-conjugated donkey antimouse (Merck Millipore; AP192P) and HRP-conjugated donkey anti-rabbit (Merck Millipore; AP182P). Membranes were then analyzed using the Western Lightning Plus Enhanced Chemiluminescence (ECL) reagent (Perkin-Elmer, Waltham, MA, USA).

Reverse transcription-PCR and gene expression analysis

Total RNA was isolated from 35-day-old mice testes using the RNeasy Mini Kit (Qiagen, Valencia, CA, USA) according to the manufacturer's protocol. cDNA synthesis was performed as described previously [20]. Primer pairs used for reverse transcription-PCR gene expression analyses are detailed in Supplementary Table S2.

\section{ChIP assay}

ChIP assays were performed as described previously [12]. Briefly, crosslinking mix (11\% formaldehyde, $100 \mathrm{~mm} \mathrm{NaCl}$, $0.5 \mathrm{~mm}$ EGTA, $50 \mathrm{~mm}$ HEPES, pH 8.0, supplemented with protease and phosphatase inhibitors) was added to testes of 35-day-old mice (ground with a pestle to disrupt their structure). Crosslinking was quenched after $15 \mathrm{~min}$ with $1.25 \mathrm{M}$ glycine and lysed with ChIP lysis buffer (1\% SDS, 10 mm EDTA, pH 8.0, $50 \mathrm{~mm}$ Tris- $\mathrm{HCl}, \mathrm{pH} 8.0$, supplemented with protease and phosphatase inhibitors) for $30 \mathrm{~min}$ at $4{ }^{\circ} \mathrm{C}$. DNA was sheared by sonication and insoluble components were removed by centrifugation. Two milligrams of protein was diluted to $600 \mathrm{ml}$ with dilution buffer ( $1 \%$ Triton X-100, $150 \mathrm{~mm} \mathrm{NaCl}, 2 \mathrm{~mm}$ EDTA, pH 8.0, 20 mm Tris-HCl, pH 8.0, supplemented with protease and phosphatase inhibitors) and precleared with protein A-Sepharose/G-Sepharose beads (Amersham Biosciences, Buckinghamshire, UK) and $4 \mu \mathrm{g}$ of salmon sperm DNA. Senataxin and CHD4 was immunoprecipitated separately using $2 \mu \mathrm{g}$ of antibody overnight at $4{ }^{\circ} \mathrm{C}$. DNA fragments were then eluted in elution buffer ( $\left.1 \% \mathrm{SDS}, 100 \mathrm{~mm} \mathrm{NaHCO}_{3}\right)$, purified and analyzed by PCR. Primer pairs used for Timpl are detailed in Supplementary Table S2. PCR products were subsequently quantitated using ImageJ 1.48v (NIH, Bethesda, MD, USA). Results for each experiment were calculated relative to the input. Error bars show the standard deviation and significance was determined using the Student's $t$-test.

\section{RLFS prediction and DRIP assay}

An RLFS was predicted (Dr Thidathip Wongsurawat, personal communication) in the 5'-UTR region of Timp1. This region was then used for DRIP analysis. Genomic DNA and RNA extraction from mice testes were performed using the DNeasy Blood and Tissue Kit (Qiagen) following the manufacturer's instructions. DRIP assay was then performed as described previously [19]. Primer pairs for Timpl are detailed in Supplementary Table S2. PCR products were subsequently quantitated using ImageJ $1.48 \mathrm{v}$. Results for each experiment were calculated relative to the input.

\section{Conflict of Interest}

The authors declare no conflict of interest.

\section{Acknowledgements}

This work was supported by the National Health and Medical Research Council (NHMRC; GNT 0631407), the Australian Research Council (ARC; DP 130100389) and the Australian Cancer Research Foundation and Cancer Institute, NSW. We acknowledge Dr Shu-Dong Zhang assistance with the connectivity mapping analysis, Dr Vladimir Kuznetsov and Dr Thidathip Wongsurawat for providing the RLFS prediction, and Dr Brent Fogel for the identification of Timp1 upregulation in microarray analyses. We acknowledge also Dr. Stephen Leppla and Prof. John Mattick who provided S9.6 antibody.

\section{References}

1 Anheim M, Monga B, Fleury $\mathrm{M}$ et al. Ataxia with oculomotor apraxia type 2: clinical, biological and genotype/phenotype correlation study of a cohort of 90 patients. Brain 2009; 132: 2688-2698.

2 Moreira MC, Klur S, Watanabe M et al. Senataxin, the ortholog of a yeast RNA helicase, is mutant in ataxia-ocular apraxia 2. Nat Genet 2004; 36: 225-227.

3 Chen YZ, Bennett CL, Huynh HM et al. DNA/RNA helicase gene mutations in a form of juvenile amyotrophic lateral sclerosis (ALS4). Am J Hum Genet 2004; 74: 1128-1135.

4 Hirano M, Quinzii CM, Mitsumoto $\mathrm{H}$ et al. Senataxin mutations and amyotrophic lateral sclerosis. Amyotroph Lateral Sc 2011; 12: 223-227.

5 Richard P, Feng S, Manley JL. A SUMO-dependent interaction between Senataxin and the exosome, disrupted in the neurodegenerative disease $\mathrm{AOA} 2$, targets the exosome to sites of transcription-induced DNA damage. Gene Dev 2013; 27: 2227-2232.

6 Kim M, Vasiljeva L, Rando OJ, Zhelkovsky A, Moore C, Buratowski S. Distinct pathways for snoRNA and mRNA termination. Mol Cell 2006; 24: 723-734.

7 Steinmetz EJ, Warren CL, Kuehner JN, Panbehi B, Ansari AZ, Brow DA. Genome-wide distribution of yeast RNA polymerase II and its control by Sen1 helicase. Mol Cell 2006; 24: 735-746.

8 Ursic D, Chinchilla K, Finkel JS, Culbertson MR. Multiple protein/protein and protein/RNA interactions suggest roles for yeast DNA/RNA helicase Sen1p in transcription, transcription-coupled DNA repair and RNA processing. Nucleic Acids Res 2004; 32: 2441-2452.

9 De Amicis A, Piane M, Ferrari F, Fanciulli M, Delia D, Chessa L. Role of senataxin in DNA damage and telomeric stability. DNA Rep 2011; 10: 199-209.

10 Lavin MF. The appropriateness of the mouse model for ataxia-telangiectasia: neurological defects but no neurodegeneration. DNA Rep 2013; 12: 612-619.

11 Suraweera A, Becherel OJ, Chen $\mathrm{P}$ et al. Senataxin, defective in ataxia oculomotor apraxia type 2 , is involved in 
the defense against oxidative DNA damage. J Cell Biol 2007; 177: 969-979.

12 Suraweera A, Lim Y, Woods R et al. Functional role for senataxin, defective in ataxia oculomotor apraxia type 2, in transcriptional regulation. Hum Mol Genet 2009; 18: 3384-3396.

13 Skourti-Stathaki K, Proudfoot NJ, Gromak N. Human senataxin resolves RNA/DNA hybrids formed at transcriptional pause sites to promote Xrn2-dependent termination. Mol Cell 2011; 42: 794-805.

14 Mischo HE, Gomez-Gonzalez B, Grzechnik P et al. Yeast Sen 1 helicase protects the genome from transcriptionassociated instability. Mol Cell 2011; 41: 21-32.

15 Aguilera A, Gomez-Gonzalez B. Genome instability: a mechanistic view of its causes and consequences. Nat Rev Genet 2008; 9: 204-217.

16 Yuce O, West SC. Senataxin, defective in the neurodegenerative disorder ataxia with oculomotor apraxia 2, lies at the interface of transcription and the DNA damage response. Mol Cell Biol 2013; 33: 406-417.

17 Alzu A, Bermejo R, Begnis M et al. Senataxin associates with replication forks to protect fork integrity across RNApolymerase-II-transcribed genes. Cell 2012; 151: 835-846.

18 Miller MS, Rialdi A, Ho JS et al. Senataxin suppresses the antiviral transcriptional response and controls viral biogenesis. Nat Immunol 2015; 16: 485-494.

19 Yeo AJ, Becherel OJ, Luff JE et al. R-loops in proliferating cells but not in the brain: implications for AOA2 and other autosomal recessive ataxias. PLOS ONE 2014; 9: e90219.

20 Becherel OJ, Yeo AJ, Stellati et al. Senataxin plays an essential role with DNA damage response proteins in meiotic recombination and gene silencing. PLoS Genet 2013; 9: e1003435.

21 Yuan L, Liu JG, Zhao J, Brundell E, Daneholt B, Hoog C. The murine SCP3 gene is required for synaptonemal complex assembly, chromosome synapsis, and male fertility. Mol Cell 2000; 5: 73-83.

22 Hecker CM, Rabiller M, Haglund K, Bayer P, Dikic I. Specification of SUMO1- and SUMO2-interacting motifs. J Biol Chem 2006; 281: 16117-16127.

23 Raman N, Nayak A, Muller S. The SUMO system: a master organizer of nuclear protein assemblies. Chromosoma 2013; 122: 475-485.

24 Vigodner M. Sumoylation precedes accumulation of phosphorylated $\mathrm{H} 2 \mathrm{AX}$ on sex chromosomes during their meiotic inactivation. Chromosome Res 2009; 17: 37-45.

25 Turner JM, Aprelikova O, Xu X et al. BRCA1, histone $\mathrm{H} 2 \mathrm{AX}$ phosphorylation, and male meiotic sex chromosome inactivation. Curr Biol 2004; 14: 2135-2142.

26 Namiki Y, Zou L. ATRIP associates with replication protein A-coated ssDNA through multiple interactions. Proc Natl Acad Sci USA 2006; 103: 580-585.

27 Shiotani B, Zou L. ATR signaling at a glance. J Cell Sci 2009; 122: 301-304.

28 Zou L, Elledge SJ. Sensing DNA damage through ATRIP recognition of RPA-ssDNA complexes. Science 2003; 300: $1542-1548$
29 Fernandez-Capetillo O, Mahadevaiah SK, Celeste et al. $\mathrm{H} 2 \mathrm{AX}$ is required for chromatin remodeling and inactivation of sex chromosomes in male mouse meiosis. Dev Cell 2003; 4: 497-508.

30 Daniel K, Lange J, Hached K et al. Meiotic homologue alignment and its quality surveillance are controlled by mouse HORMAD1. Nat Cell Biol 2011; 13: 599-U232.

31 Liu SQ, Opiyo SO, Manthey K et al. Distinct roles for DNA-PK, ATM and ATR in RPA phosphorylation and checkpoint activation in response to replication stress. Nucleic Acids Res 2012; 40: 10780-10794.

32 Yilmaz S, Sancar A, Kemp MG. Multiple ATR-Chk1 pathway proteins preferentially associate with checkpointinducing DNA substrates. PLoS Ine 2011; 6: e22986.

33 Cha RS, Kleckner N. ATR homolog Mec1 promotes fork progression, thus averting breaks in replication slow zones. Science 2002; 297: 602-606.

34 Sorensen CS, Syljuasen RG. Safeguarding genome integrity: the checkpoint kinases ATR, CHK1 and WEE1 restrain CDK activity during normal DNA replication. Nucleic Acids Res 2012; 40: 477-486.

35 Liu S, Ho CK, Ouyang J, Zou L. Nek1 kinase associates with ATR-ATRIP and primes ATR for efficient DNA damage signaling. Proc Natl Acad Sci USA 2013; 110: 2175-2180.

36 Xue YT, Wong JM, Moreno GT, Young MK, Cote J, Wang WD. NURD, a novel complex with both ATPdependent chromatin-remodeling and histone deacetylase activities. Mol Cell 1998; 2: 851-861.

37 McArt DG, Zhang SD. Identification of candidate smallmolecule therapeutics to cancer by gene-signature perturbation in connectivity mapping. PLOS ONE 2011; 6: e16382.

38 Schmidt DR, Schreiber SL. Molecular association between ATR and two components of the nucleosome remodeling and deacetylating complex, HDAC2 and CHD4. Biochemistry-Us 1999; 38: 14711-14717.

39 Urquhart AJ, Gatei M, Richard DJ, Khanna KK. ATM mediated phosphorylation of CHD4 contributes to genome maintenance. Genome Integr 2011; 2 : 1.

40 Musselman CA, Ramirez J, Sims JK et al. Bivalent recognition of nucleosomes by the tandem PHD fingers of the CHD4 ATPase is required for CHD4-mediated repression. Proc Natl Acad Sci USA 2012; 109: 787-792.

41 Morris SA, Baek S, Sung $\mathrm{MH}$ et al. Overlapping chromatin-remodeling systems collaborate genome wide at dynamic chromatin transitions. (vol 21, pg 73, 2014). Nat Struct Mol Biol 2014; 21: 1106-1106.

42 Page $\mathrm{J}$, de la Fuente $\mathrm{R}$, Manterola $\mathrm{M}$ et al. Inactivation or non-reactivation: what accounts better for the silence of sex chromosomes during mammalian male meiosis? Chromosoma 2012; 121: 307-326.

43 Ginno PA, Lott PL, Christensen HC, Korf I, Chedin F. R-loop formation is a distinctive characteristic of unmethylated human CpG island promoters. Mol Cell 2012; 45: 814-825.

44 Zhang Y, Reinberg D. Transcription regulation by histone methylation: interplay between different covalent modifications of the core histone tails. Genes Dev 2001; 15: 2343-2360. 
45 An W. Histone acetylation and methylation: combinatorial players for transcriptional regulation. Subcell Biochem 2007; 41: 351-369.

46 Ramirez J, Dege C, Kutateladze TG, Hagman J. MBD2 and multiple domains of CHD4 are required for transcriptional repression by $\mathrm{Mi}-2 / \mathrm{NuRD}$ complexes. Mol Cell Biol 2012; 32: 5078-5088.

47 Royo H, Prosser H, Ruzankina Y et al. ATR acts stage specifically to regulate multiple aspects of mammalian meiotic silencing. Gene Dev 2013; 27: 1484-1494.

48 Nam EA, Cortez D. AIR signalling: more than meeting at the fork. Biochem J 2011; 436: 527-536.

49 Zeman MK, Cimprich KA. Causes and consequences of replication stress. Nat Cell Biol 2014; 16: 2-9.

50 Refolio E, Cavero S, Marcon E, Freire R, San-Segundo PA. The Ddc2/ATRIP checkpoint protein monitors meiotic recombination intermediates. $J$ Cell Sci 2011; 124: 2488-2500.

51 Wu CS, Ouyang J, Mori E et al. SUMOylation of ATRIP potentiates DNA damage signaling by boosting multiple protein interactions in the ATR pathway. Gene Dev 2014; 28: $1472-1484$.

52 Xiao Z, Chang JG, Hendriks IA, Sigurethsson JO, Olsen JV, Vertegaal AC. System-wide analysis of SUMOylation dynamics in response to replication stress reveals novel small ubiquitin-like modified target proteins and acceptor lysines relevant for genome stability. Mol Cell Proteom 2015; 14: 1419-1434.

53 Levy MA, Kernohan KD, Jiang Y, Berube NG. ATRX promotes gene expression by facilitating transcriptional elongation through guanine-rich coding regions. Hum $\mathrm{Mol}$ Genet 2014; 24: 1824-1835.

54 Haeusler AR, Donnelly CJ, Periz G et al. C9orf72 nucleotide repeat structures initiate molecular cascades of disease. Nature 2014; 507: 195-200.
55 Mosallanejad K, Sekine Y, Ishikura-Kinoshita S et al. The DEAH-box RNA helicase DHX15 activates NF-kappaB and MAPK signaling downstream of MAVS during antiviral responses. Sci Signal 2014; 7: ra40.

56 Magalska A, Schellhaus AK, Moreno-Andres D et al. RuvB-like ATPases function in chromatin decondensation at the end of mitosis. Dev Cell 2014; 31: 305-318.

57 Ahringer J. NuRD and SIN3 histone deacetylase complexes in development. Trends Genet 2000; 16: 351-356.

58 O'Shaughnessy A, Hendrich B. CHD4 in the DNA-damage response and cell cycle progression: not so NuRDy now. Biochem Soc Trans 2013; 41: 777-782.

59 Larsen DH, Poinsignon C, Gudjonsson $\mathrm{T}$ et al. The chromatin-remodeling factor CHD4 coordinates signaling and repair after DNA damage. J Cell Biol 2010; 190: 731-740.

60 Cheng J, Blum R, Bowman C et al. A role for $\mathrm{H} 3 \mathrm{~K} 4$ monomethylation in gene repression and partitioning of chromatin readers. Mol Cell 2014; 53: 979-992.

61 Sollier J, Stork CT, Garcia-Rubio ML, Paulsen RD, Aguilera A, Cimprich KA. Transcription-coupled nucleotide excision repair factors promote R-loop-induced genome instability. Mol cell 2014; 56: 777-785.

(Supplementary information is linked to the online version of the paper on the Cell Discovery website.)

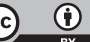

This work is licensed under a Creative Commons Attribution 4.0 International License. The images or other third party material in this article are included in the article's Creative Commons license, unless indicated otherwise in the credit line; if the material is not included under the Creative Commons license, users will need to obtain permission from the license holder to reproduce the material. To view a copy of this license, visit http://creativecommons.org/licenses/by/4.0/ 